Subscriber access provided by Caltech Library

\title{
Article
}

\section{Isocanthine Synthesis via Rh(III)-Catalyzed Intramolecular C\#H Functionalization}

Anthony Y. Chen, Qianqian Lu, Yao Fu, Richmond Sarpong, Brian M. Stoltz, and Haiming Zhang

J. Org. Chem., Just Accepted Manuscript • DOI: 10.1021/acs.joc.7b02731 • Publication Date (Web): 28 Nov 2017

Downloaded from http://pubs.acs.org on November 29, 2017

\section{Just Accepted}

"Just Accepted" manuscripts have been peer-reviewed and accepted for publication. They are posted online prior to technical editing, formatting for publication and author proofing. The American Chemical Society provides "Just Accepted" as a free service to the research community to expedite the dissemination of scientific material as soon as possible after acceptance. "Just Accepted" manuscripts appear in full in PDF format accompanied by an HTML abstract. "Just Accepted" manuscripts have been fully peer reviewed, but should not be considered the official version of record. They are accessible to all readers and citable by the Digital Object Identifier (DOI®). "Just Accepted" is an optional service offered to authors. Therefore, the "Just Accepted" Web site may not include all articles that will be published in the journal. After a manuscript is technically edited and formatted, it will be removed from the "Just Accepted" Web site and published as an ASAP article. Note that technical editing may introduce minor changes to the manuscript text and/or graphics which could affect content, and all legal disclaimers and ethical guidelines that apply to the journal pertain. ACS cannot be held responsible for errors or consequences arising from the use of information contained in these "Just Accepted" manuscripts. 


\section{INTRODUCTION}

Isocanthines are a class of tetracyclic $\gamma$-carbolines that have demonstrated widespread clinical use as cardiovascular agents, ${ }^{1}$ antiemetic $5-\mathrm{HT}_{3}$ receptor antagonists for chemotherapy patients, ${ }^{2}$ as well as potential treatments for CNS disorders. ${ }^{3}$ Surprisingly, very few syntheses of isocanthines (Scheme 1) have been reported. Typically the reported syntheses employ either thermal cyclization of a 1azatriene $^{4}$ (Scheme 1, A) or intramolecular hetero-Diels-Alder cycloaddition $^{5}$ of an alkyne-tethered indole oxime (Scheme 1, B). ${ }^{6}$ Unfortunately, these syntheses suffer from very limited scope, the requirement of high reaction temperatures, or low yield over multiple steps. ${ }^{4,6}$ Larock's isocanthine synthesis ${ }^{7}$ employing a Pd-catalyzed intramolecular iminoannulation affords excellent yields with a wide functional group tolerance, but requires pre-installation of a halide, thus is not very efficient (Scheme 1, C). Therefore, a more direct synthesis of isocanthines is highly desirable.

Recently, Rh-catalyzed $\mathrm{C}-\mathrm{H}$ functionalization reactions ${ }^{8}$ have attracted much attention in the literature and have been employed in synthesizing an array of interesting heterocycles, such as indoles, ${ }^{9}$ isoquinolines, ${ }^{10,11}$ isoquinolones, ${ }^{12}$ pyrroles, ${ }^{13}$ pyridines $^{14}$ and polyheterocycles. ${ }^{15}$ Inspired by Fagnou's isoquinoline synthesis ${ }^{10}$ from aryl aldimines and alkynes (eq 1), we envisioned that isocanthines could readily be synthesized by $\mathrm{C}-\mathrm{H}$ functionalization of alkyne-tethered indole tert-

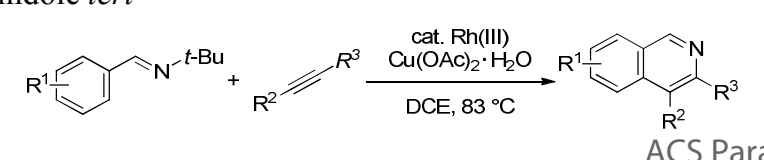

butylimines (Scheme 1, D). Herein, we wish to report an efficient synthesis of substituted isocanthines via intramolecular $\mathrm{Rh}(\mathrm{III})$-catalyzed $\mathrm{C}-\mathrm{H}$ functionalization of alkyne-tethered indoles.

Scheme 1. Synthetic strategies to Isocanthines

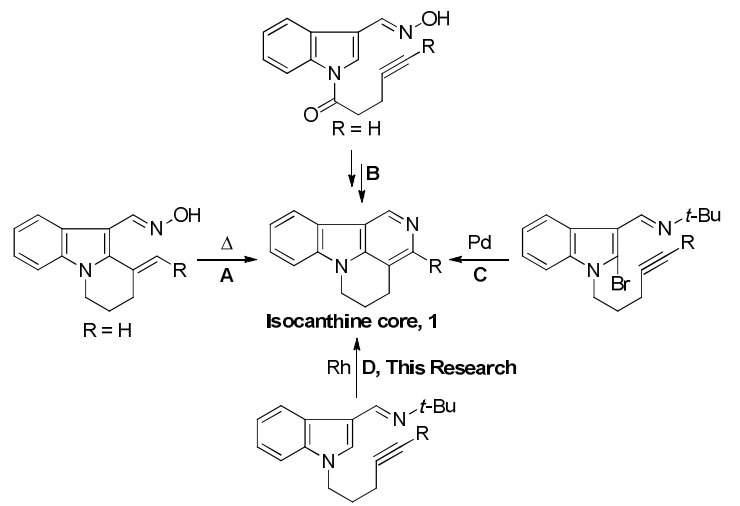

\section{RESULTS AND DISCUSSION}

Our investigation commenced with the optimization of 3-nbutyl-isocanthine (1a) formation from imine 2a by screening a variety of catalyst and oxidant systems (Table 1). The optimal "standard conditions" employed $2.5 \mathrm{~mol} \%$ tris(acetonitrile)pentamethylcyclopentadienylrhodium(III) hexafluoroantimonate $(\mathbf{C 1})^{16}$ as the catalyst, 2.1 equiv of $\mathrm{Cu}(\mathrm{OAc})_{2}$ as the oxidant and the reaction was carried out in dichloroethane (DCE) in a sealed vial at $100{ }^{\circ} \mathrm{C}$ for $16 \mathrm{~h}$. Under this set of optimal conditions, the reaction afforded $93 \%$ conversion and $73 \%$ assay yield based on quantitative 
HPLC analysis. The desired product (1a) was subsequently isolated in $73 \%$ yield (Table 1 , entry 1 ). When the reaction was performed at a lower temperature $\left(83{ }^{\circ} \mathrm{C}\right)$, much lower conversion and assay yield were obtained (Table 1, entry 2). Without Rh complex $\mathbf{C 1}$, the reaction did not produce any desired product 1a, which indicates that formation of the isocanthine did not proceed via simple thermal hetero-DielsAlder cycloaddition and subsequent oxidative aromatization (Table 1, entry 3). Lowering the loading of C1 to $1 \mathrm{~mol} \%$ resulted in low conversion and assay yield of product $\mathbf{1 a}$ (Table 1, entry 4). When $\left[\mathrm{Cp}^{*} \mathrm{RhCl}_{2}\right]_{2}(\mathbf{C 2})$ and $[\mathrm{Rh}(\mathrm{COD}) \mathrm{Cl}]_{2}$ (C3) were employed, both reactions proceeded with inferior conversion relative to the optimal conditions (Table 1, entries 5-6). Palladium catalysts, such as palladium(II) acetate (C4) and palladium(II) trifluoroacetate (C5), did not generate significant conversion (Table 1, entries 7-8). Among the oxidants that were screened, $\mathrm{Cu}(\mathrm{OAc})_{2} \cdot \mathrm{H}_{2} \mathrm{O}$ which was used in Fagnou's isoquinoline synthesis ${ }^{10}$ resulted in $86 \%$ conversion and $68 \%$ assay yield of the desired product (Table 1 , entry 9). The lower yield was possibly due to partial hydrolysis of imine $\mathbf{2 a}$ to the corresponding aldehyde under the reaction conditions since about $8 \%$ of the aldehyde was observed upon reaction completion based on HPLC analysis as opposed to $<2 \%$ of the aldehyde when anhydrous $\mathrm{Cu}(\mathrm{OAc})_{2}$ was employed. Other oxidants, for example, silver acetate, benzoquinone and (diacetoxyiodo)benzene all produced low assay yield of product 1a (Table 1, entries 10-12).

Table 1. Optimization of Isocanthine 1a Formation ${ }^{a}$

$\begin{array}{clcc}\text { variation from the "standard" } & \begin{array}{c}\text { conv } \\ (\%)^{b}\end{array} & \begin{array}{c}\text { yield } \\ (\%)^{c}\end{array} \\ \text { entry } & \text { conditions } & 93 & 73(73) \\ 1 & \text { None } & 53 & 39 \\ 2 & 83{ }^{\circ} \mathrm{C} \text { instead of } 100{ }^{\circ} \mathrm{C} & 0 & 0 \\ 3 & \text { no } \mathbf{C 1} & 20 & 19 \\ 4 & \mathbf{C 1}(1 \mathrm{~mol} \%) \text { as catalyst } & 29 & 25 \\ 5 & \mathbf{C 2}(1.25 \text { mol \%) as catalyst } & <5 & <5 \\ 6 & \mathbf{C 3}(1.25 \text { mol \%) as catalyst } & <5 & <5 \\ 7 & \mathbf{C 4} \text { as catalyst } & <5 & <5 \\ 8 & \mathbf{C 5} \text { as catalyst } & 86 & 68 \\ 9 & \mathrm{Cu}(\mathrm{OAc})_{2} \cdot \mathrm{H}_{2} \mathrm{O} \text { as oxidant } & 30 & 27 \\ 10 & \mathrm{AgOAc} \text { as oxidant } & 50 & <5 \\ 11 & \text { benzoquinone as oxidant } & 61 & <5 \\ 12 & \mathrm{PhI}(\mathrm{OAc})_{2} \text { as oxidant } & \end{array}$

$\left[\mathrm{Cp}{ }^{*} \mathrm{Rh}(\mathrm{MeCN})_{3}\right]\left(\mathrm{SbF}_{6}\right)_{2} \quad\left[\begin{array}{llll}\left.\mathrm{Cp}^{*} \mathrm{RhCl}_{2}\right]_{2} & {\left[\mathrm{Rh}(\mathrm{COD}) \mathrm{Cl}_{2}\right.} & \mathrm{Pd}(\mathrm{OAC})_{2} & \mathrm{Pd}(\mathrm{TFA})_{2}\end{array}\right.$ $\begin{array}{llllll}C 1 & C 2 & C 3 & C 4 & C 5\end{array}$

${ }^{a}$ All reactions were performed using $0.50 \mathrm{mmol}$ of $\mathbf{2 a}$ in DCE (3.0 $\mathrm{mL}$ ) in sealed vials for $16 \mathrm{~h} .{ }^{b}$ Determined by HPLC analysis. ${ }^{c}$ Assay yields determined by quantitative HPLC analysis. The number in parentheses is the isolated yield.

We next examined the substrate scope and limitations of this $\mathrm{Rh}(\mathrm{III})$-catalyzed isocanthine synthesis. It is worth mentioning that the transformation of the aldehydes to the corresponding tert-butylimines is essentially quantitative, thus requiring no further purification and characterization of the starting imines used for the subsequent $\mathrm{C}-\mathrm{H}$ functionalization.
Indeed, the one-pot imine formation/C-H functionalization process employing aldehyde $\mathbf{3 a}$ afforded the same isolated yield $(73 \%)$ as that of the step-wise approach (Table 2, entry 1). Therefore, by employing a one-pot protocol, namely imine formation, followed by $\mathrm{Rh}(\mathrm{III})$-catalyzed intramolecular $\mathrm{C}-\mathrm{H}$ functionalization, we were able to synthesize a variety of substituted isocanthines (Table 2).

The electronic effects of the substituents on the indole ring were first examined. Gratifyingly, both electron-donating and withdrawing substituents are well tolerated on the 5-position of indole. For example, electron-donating methyl and methoxy substituted indoles $\mathbf{3 b}$ and $\mathbf{3} \mathbf{c}$ produced the desired isocanthines (1b and 1c) in $78 \%$ and $75 \%$ yields, respectively (Table 2, entries 2-3). Indole 3d substituted with an electronwithdrawing fluorine atom afforded isocanthine $\mathbf{1 d}$ in an excellent $90 \%$ yield (Table 2, entry 4 ). 5-Bromo-substituted indole $3 \mathbf{e}$ also participated in this $\mathrm{C}-\mathrm{H}$ functionalization reaction, generating a moderate yield $(40 \%)$ of the desired bromoisocanthine 1e. (Table 2, entry 5). As expected, indoles substituted with either electron-donating $(\mathrm{MeO}, 3 \mathbf{3 f})$ or electron-withdrawing $\left(\mathrm{CO}_{2} \mathrm{Me}, \mathbf{3 g}\right)$ groups at the 6-position uneventfully gave the desired products $\mathbf{1 f}$ and $\mathbf{1 g}$ in $78 \%$ and $82 \%$, respectively (Table 2 , entries $6-7$ ).

Table 2. Scope of Isocanthine Formation ${ }^{a}$

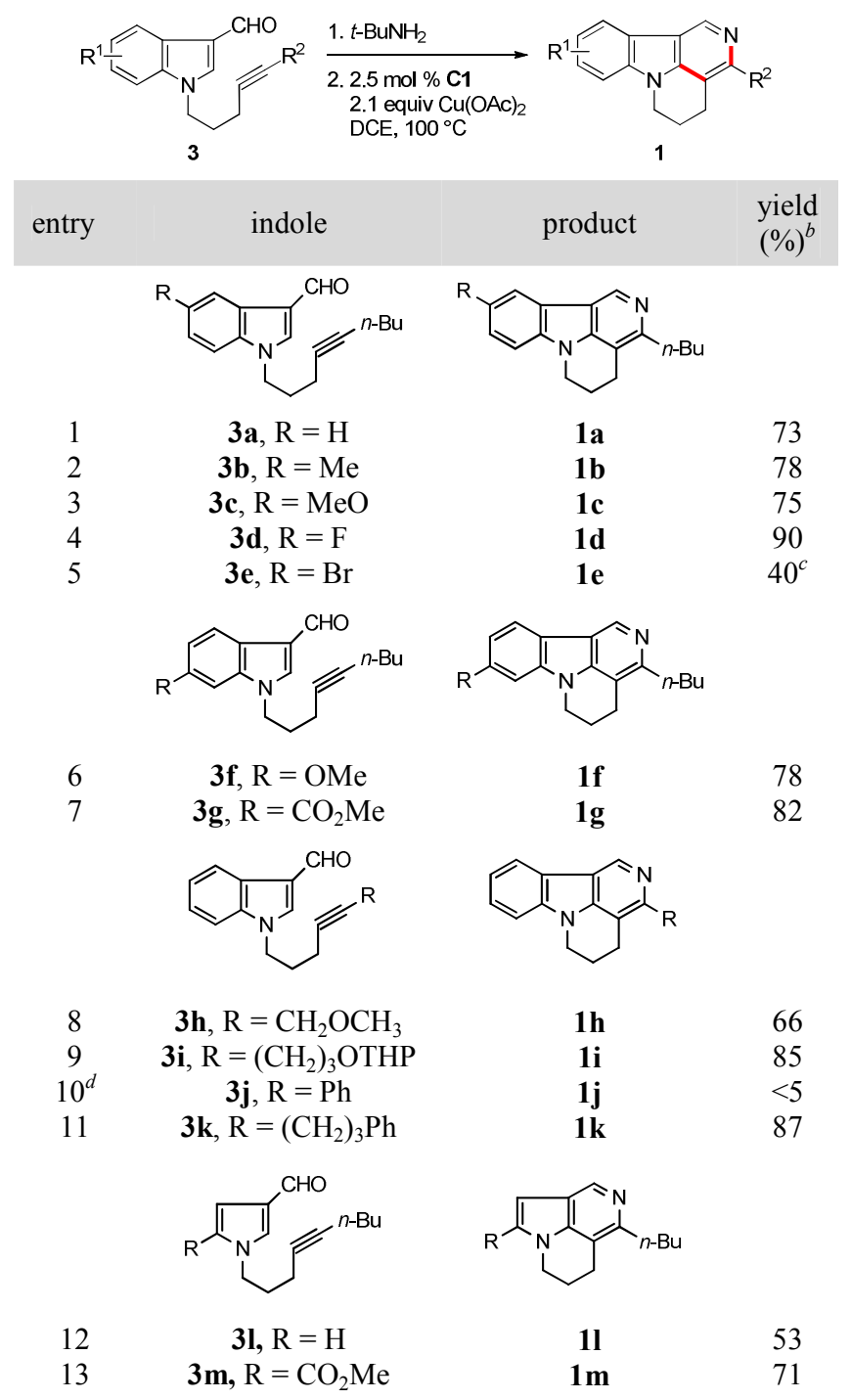


${ }^{a}$ All reactions were performed using $0.50 \mathrm{mmol}$ of $\mathbf{3}$ in $t-\mathrm{BuNH}_{2}$ $(3.0 \mathrm{~mL})$ at $80{ }^{\circ} \mathrm{C}$ for $2 \mathrm{~h}$, followed by $\left[\mathrm{Cp} * \mathrm{Rh}(\mathrm{MeCN})_{3}\right]\left(\mathrm{SbF}_{6}\right)_{2}$ $(\mathbf{C 1}, 2.5 \mathrm{~mol} \%), \mathrm{Cu}(\mathrm{OAc})_{2}(2.1$ equiv) in DCE $(3.0 \mathrm{~mL})$ at 100 ${ }^{\circ} \mathrm{C}$ in sealed vials for $16 \mathrm{~h} .{ }^{b}$ Isolated yield. ${ }^{c} 30 \mathrm{~A} \%$ of isocanthine 1a was observed by HPLC. ${ }^{d}$ Reaction performed at $150{ }^{\circ} \mathrm{C}$.

The substituent effect on the alkyne was then investigated. Indoles with ether substituted alkynes underwent the intramolecular annulation smoothly, giving the desired isocanthine products (1h and $\mathbf{1 i}$ ) in good yields (Table 2, entries 8-9). Surprisingly, indole $\mathbf{3 j}$ tethered with a phenylsubstituted alkyne failed to produce isocanthine product $\mathbf{1} \mathbf{j}$ even at an elevated temperature $150{ }^{\circ} \mathrm{C}$ (Table , entry 10). ${ }^{17}$ However, indole 3k tethered with a phenylpropyl-substituted alkyne uneventfully afforded $87 \%$ yiled of isocanthine $1 \mathbf{k}$ (Table 2, entry 11). This isocanthine synthesis could also be extended to alkyne-tethered pyrroles for the synthesis of annulated 5-azadindoles. In fact, pyrroles $\mathbf{3 l}$ and $\mathbf{3 m}$ were subjected to the standard reaction conditions, producing 53\% and $71 \%$ yields of the desired 5-azaindoles (1l and $\mathbf{1 m}$ ), respectively (Table 2, entries 12-13). Thus, one can envision that this chemistry could be further expanded into a general method for the synthesis of a wide spectrum of annulated 5azaindoles.

To further demonstrate the synthetic utility of the brominefunctionalized isocanthine, 1d was converted to cyclopropyland 2-furyl-substituted derivatives $\mathbf{4 a - b}$ in moderate to good yields via Suzuki-Miyaura cross-coupling reactions (Scheme 2). ${ }^{18}$ The strucure of isocanthine $\mathbf{4 b}$ was established unambiguously by single-crystal X-ray diffraction analysis.

\section{Scheme 2. Synthetic Utility of Bromoisocanthine $1 \mathrm{~d}^{a}$}
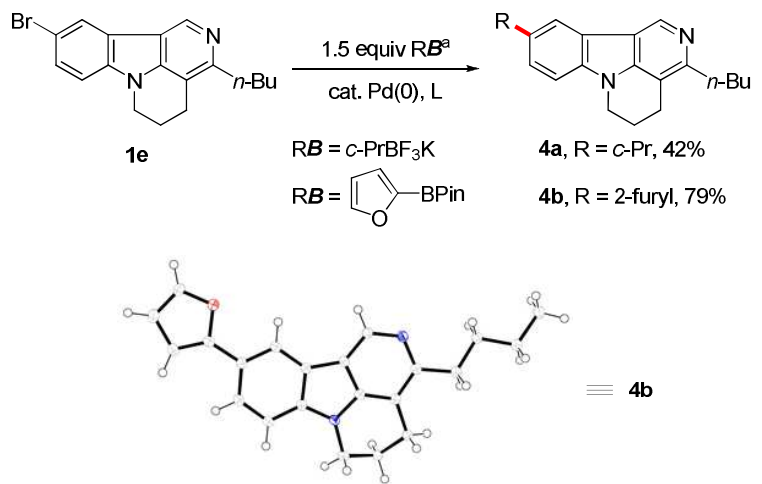

${ }^{a}$ Conditions: (4a), $\mathrm{Pd}(\mathrm{OAc})_{2}$ (10 mol \%), di(1-adamanyl)- $n$ butylphosphine (15 mol \%), $\mathrm{Cs}_{2} \mathrm{CO}_{3}$ (3.0 equiv), $c-\mathrm{PrBF}_{3} \mathrm{~K}(1.5$ equiv), $\mathrm{PhMe}: \mathrm{H}_{2} \mathrm{O}(10: 1), 100{ }^{\circ} \mathrm{C}$. (4b), $\mathrm{Pd}(\mathrm{dtbpp}) \mathrm{Cl}_{2}(5 \mathrm{~mol} \%)$, 2-furyl pinacol ester (1.5 equiv), $\mathrm{K}_{3} \mathrm{PO}_{4} \cdot \mathrm{H}_{2} \mathrm{O}$ (2 equiv), THF: $\mathrm{H}_{2} \mathrm{O}$ $(5: 1), 65^{\circ} \mathrm{C}$.

We conducted DFT calculations using imine 2a as an example to assist in understanding the mechanism of this $\mathrm{C}-\mathrm{H}$ functionalization reaction. The free energy profile of the $\mathrm{Rh}(\mathrm{III}) / \mathrm{Rh}(\mathrm{I})$ catalytic cycle begining from imine 2a and rhodaycle Rh0 is shown in Figure 1. In accord with literature precedent, ${ }^{10}$ the mechanism involves an initial imine coordination of $\mathbf{2 a}$ and rhodacycle $\mathbf{R h \mathbf { 0 }}$ to form rhodacycle Int1, followed by ortho-directed $\mathrm{C}-\mathrm{H}$ functionalization via concerted metalation deprotonation, ${ }^{9,12 a}$ (via Int2), then alkyne coordination (via Int3) and insertion to afford sevenmembered rhodacycle Int4. TS1 and TS2 are the transition states for these two steps, and have barriers of 16.4 and 9.2 $\mathrm{kcal} / \mathrm{mol}$, respectively. Rhodacycle Int4 then undergoes reductive elimination via TS3 with a barrier of $17.2 \mathrm{kcal} / \mathrm{mol}$ to produce intermediate Int5. Int5 then proceeds with tertbutyl fragmentation and catalyst regeneration in the presence of 2 equivalents of $\mathrm{Cu}(\mathrm{OAc})_{2}$ to generate the isocanthine product 1a, along with byproducts isobutene, acetic acid and $\mathrm{Cu}(\mathrm{OAc})$, and catalyst $\mathbf{R h} \mathbf{0}$.

\section{CONCLUSIONS}

In summary, we have developed a $\mathrm{C}-\mathrm{H}$ functionalization approach to substituted isocanthines from alkyne-tethered indole-3-carboxaldehydes and tert-butylamine using $2.5 \mathrm{~mol}$ $\%$ of $\left[\mathrm{Cp} * \mathrm{Rh}(\mathrm{MeCN})_{3}\right]\left(\mathrm{SbF}_{6}\right)_{2}$ as the catalyst and 2.1 equiv of $\mathrm{Cu}(\mathrm{OAc})_{2}$ as the oxidant in DCE at $100{ }^{\circ} \mathrm{C}$. Both electrondonating and electron-withdrawing substituents are tolerated on the 5- and 6-positions of the indole ring. This chemistry can also be extended to pyrrole derivatives for the synthesis of annulated 5-azaindoles. Bromine substitution on the indole ring allows for further functionalization of the isocanthine framework via Suzuki-Miyaura cross-coupling reactions. Theoretical calculations suggest that the mechanism of this chemistry involves ortho-directed $\mathrm{C}-\mathrm{H}$ functionalization via a concerted metalation deprotonation pathway, followed by alkyne coordination and insertion, then reductive elimination and tert-butyl fragmentation to afford the desired isocanthine product. 

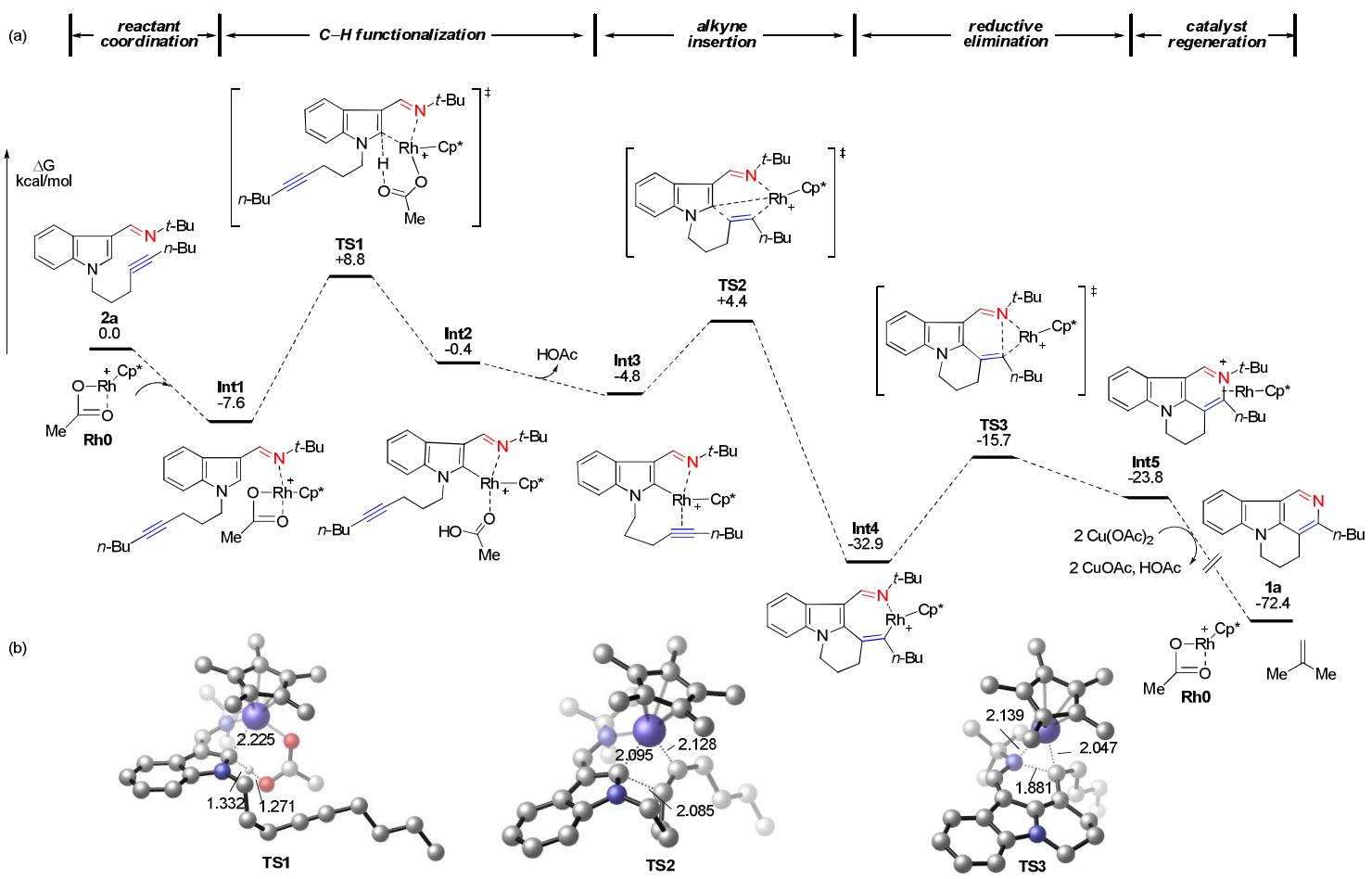

Figure 1. (a) Free energy profiles for the mechanism of Rh(III)-catalyzed formation of isocanthine 1a. (b) Computed configurations of transition states with selected bond distances shown in angstroms $(\AA)$. Some hydrogen atoms are omitted for clarity.

\section{EXPERIMENTAL SECTION}

\section{Materials and Methods}

Unless stated otherwise, reactions were performed in 4-dram vials sealed with Teflon-lined caps. Commercially obtained solvents and reagents were used as received. Thin-layer chromatography (TLC) was performed using glass-backed plates pre-coated with EMD silica gel $60 \mathrm{~F}_{254}$ and visualized using UV light $(254 \mathrm{~nm}) .{ }^{1} \mathrm{H}$ and ${ }^{13} \mathrm{C}$ NMR spectra were recorded on 300 or $400 \mathrm{MHz}$ Bruker spectrometer and chemical shifts are reported relative to the residual solvent peak $\left({ }^{1} \mathrm{H} \mathrm{NMR}, \delta 7.26\right.$ for $\mathrm{CDCl}_{3},{ }^{13} \mathrm{C} \mathrm{NMR}, \delta 77.0$ for $\left.\mathrm{CDCl}_{3}\right) .{ }^{1} \mathrm{H}$ NMR spectral data are reported in terms of: chemical shift $(\delta \mathrm{ppm})$, multiplicity, coupling constant $(\mathrm{Hz})$, and integration. ${ }^{13} \mathrm{C}$ NMR spectral data are reported in terms of chemical shift $(\delta$ ppm). Flash column chromatography was performed using a CombiFlash ISCO instrument, using pre-packed RediSep silica gel columns. HPLC analyses were performed using an Agilent 1290 Infinity Series HPLC instrument. IR spectra were recorded using a Bruker Alpha Platinum-ATR spectrometer and are reported in wavenumbers $\left(\mathrm{cm}^{-1}\right)$. HRMS data were obtained using a LTQ Orbitrap Discovery (Thermo Fisher Scientific) at Genentech, Inc. Melting points were measured on a Büchi Melting Point B-540 apparatus.

\section{General Experimental Procedure}

(8-Chlorooct-4-yn-1-yl)benzene: To a flame-dried $50 \mathrm{~mL}$ round-bottom flask was added 5-chloropent-1-yne (0.968 g, 9.44 $\mathrm{mmol})$, followed by THF $(9.4 \mathrm{~mL})$, and the mixture was cooled to $78{ }^{\circ} \mathrm{C}$ under $\mathrm{N}_{2} . n$-Butyllithium solution $(2.5 \mathrm{M}$ in hexanes, $4.2 \mathrm{~mL}$, 1.1 equiv) was then added dropwise, and the mixture was stirred at $-78{ }^{\circ} \mathrm{C}$ for $30 \mathrm{~min}$ before dropwise addition of (3iodopropyl)benzene (1.5 mL, $9.44 \mathrm{mmol}, 1.0$ equiv). The mixture was stirred at $-78^{\circ} \mathrm{C}$ for $30 \mathrm{~min}$, warmed to $23{ }^{\circ} \mathrm{C}$ and stirred at 23 ${ }^{\circ} \mathrm{C}$ for $16 \mathrm{~h}$. The reaction was quenched with saturated aqueous ammonium chloride and the aqueous layer was extracted with ethyl acetate $(10 \mathrm{~mL}, \times 3)$. The combined organic layers were washed with brine $(10 \mathrm{~mL})$, dried over sodium sulfate $(2 \mathrm{~g})$, concentrated in vacuum and purified by silica gel column chromatography using ethyl acetate in hexanes $(0-2 \%)$ to afford (8-chlorooct-4-yn-1yl)benzene as a colorless oil $\left(1.27 \mathrm{~g}, 61 \%\right.$ ); FTIR (thin film, $\mathrm{cm}^{-1}$ )
$3026,2940,2859,1603,1453 ;{ }^{1} \mathrm{H}$ NMR $\left(400 \mathrm{MHz}, \mathrm{CDCl}_{3}\right) \delta 7.32$ -7.26 (m, 2H), 7.19 (ddd, $J=6.1,2.1,1.2 \mathrm{~Hz}, 3 \mathrm{H}), 3.67$ (t, $J=6.4$ $\mathrm{Hz}, 2 \mathrm{H}), 2.75-2.67(\mathrm{~m}, 2 \mathrm{H}), 2.37(\mathrm{tt}, J=6.7,2.4 \mathrm{~Hz}, 2 \mathrm{H}), 2.17(\mathrm{tt}$, $J=7.1,2.4 \mathrm{~Hz}, 2 \mathrm{H}), 1.95$ (quintet, $J=6.5 \mathrm{~Hz}, 2 \mathrm{H}), 1.87-1.75(\mathrm{~m}$, $2 \mathrm{H}) ;{ }^{13} \mathrm{C}$ NMR $\left(101 \mathrm{MHz}, \mathrm{CDCl}_{3}\right) \delta 141.9,128.7,128.5,126.0$, $81.1,78.8,44.0,35.0,31.9,30.8,18.3,16.4$; HRMS (ESI-TOF) $\mathrm{m} / \mathrm{z}$ : $[\mathrm{M}+\mathrm{H}]^{+}$calcd for $\mathrm{C}_{14} \mathrm{H}_{18} \mathrm{Cl} 221.1097$; Found, 221.1071.

\section{Synthesis of $\boldsymbol{N}$-Alkylated Indole-3-carboxaldehydes}

To a $20 \mathrm{~mL}$ vial was added indole-3-carboxaldehyde, sodium iodide ( 1.5 equiv), cesium carbonate (1.5 equiv), and chloroalkyne (1.2 equiv) in acetonitrile $(10 \mathrm{~mL} / \mathrm{g})$. The vial was then sealed and heated to $80^{\circ} \mathrm{C}$. After $16 \mathrm{~h}$, solids were filtered off and the cake was washed with acetonitrile $(2 \mathrm{~mL} / \mathrm{g}, \times 3)$. The combined organic solution was concentrated and purified by silica gel column chromatography with ethyl acetate in hexanes gradient eluent to afford the desired $N$-alkylated indole-3-carboxaldehyde 3 .

1-(Non-4-yn-1-yl)-1 $\boldsymbol{H}$-indole-3-carbaldehyde (3a): $1 H$-indole3-carbaldehyde $(1.00 \mathrm{~g}, 6.89 \mathrm{mmol})$, sodium iodide $(1.55 \mathrm{~g}, 1.5$ equiv), cesium carbonate (3.37 g, 1.5 equiv), 1-chloronon-4-yne $(1.31 \mathrm{~g}, 1.2$ equiv) and acetonitrile $(10.0 \mathrm{~mL})$ were employed. Upon silica gel column chromatography purification using ethyl acetate in hexanes $(0-50 \%)$, compound 3a was isolated as a yellow oil (1.79 g, 97\%). FTIR (thin film, $\mathrm{cm}^{-1}$ ) 2956, 2930, 1655, 1531, 1467; ${ }^{1} \mathrm{H}$ NMR $\left(300 \mathrm{MHz}, \mathrm{CDCl}_{3}\right) \delta 10.02(\mathrm{~s}, 1 \mathrm{H}), 8.35-8.28(\mathrm{~m}, 1 \mathrm{H}), 7.77$ (s, 1H), $7.47-7.28(\mathrm{~m}, 3 \mathrm{H}), 4.35(\mathrm{t}, J=6.7 \mathrm{~Hz}, 2 \mathrm{H}), 2.26-2.15$ $(\mathrm{m}, 4 \mathrm{H}), 2.04$ (quintet, $J=6.4 \mathrm{~Hz}, 2 \mathrm{H}), 1.58-1.38(\mathrm{~m}, 4 \mathrm{H}), 0.94(\mathrm{t}$, $J=7.1 \mathrm{~Hz}, 3 \mathrm{H}) ;{ }^{13} \mathrm{C}$ NMR $\left(75 \mathrm{MHz}, \mathrm{CDCl}_{3}\right) \delta 184.4,138.9,137.2$, $125.4,123.9,122.8,122.0,118.0,110.2,82.3,77.8,45.6,31.1$ 28.7, 22.0, 18.4, 16.0, 13.7; HRMS (ESI-TOF) $\mathrm{m} / \mathrm{z}:[\mathrm{M}+\mathrm{H}]^{+}$calcd for $\mathrm{C}_{18} \mathrm{H}_{22} \mathrm{NO} 268.1701$; Found, 268.1703.

5-Methyl-1-(non-4-yn-1-yl)-1 $\boldsymbol{H}$-indole-3-carbaldehyde (3b): 5methyl- $1 H$-indole-3-carbaldehyde (500 mg, $3.14 \mathrm{mmol}$ ), sodium iodide ( $471 \mathrm{mg}, 1.5$ equiv), cesium carbonate ( $1.54 \mathrm{~g}, 1.5$ equiv), 1 chloronon-4-yne (598 mg, 1.2 equiv) and acetonitrile $(5.0 \mathrm{~mL})$ were employed. Upon silica gel column chromatography purification using ethyl acetate in hexanes $(0-50 \%)$, compound $\mathbf{3 b}$ was isolated as a yellow oil (857 mg, 97\%). FTIR (thin film, $\mathrm{cm}^{-1}$ ) 2954, 2930, $1652,1528,1485 ;{ }^{1} \mathrm{H}$ NMR $\left(300 \mathrm{MHz}, \mathrm{CDCl}_{3}\right) \delta 9.98(\mathrm{~s}, 1 \mathrm{H}), 8.12$ (s, 1H), $7.72(\mathrm{~s}, 1 \mathrm{H}), 7.31(\mathrm{~d}, J=8.4 \mathrm{~Hz}, 1 \mathrm{H}), 7.16(\mathrm{dd}, J=8.4,1.4$ 
$\mathrm{Hz}, 1 \mathrm{H}), 4.32(\mathrm{t}, J=6.7 \mathrm{~Hz}, 2 \mathrm{H}), 2.49(\mathrm{~s}, 3 \mathrm{H}), 2.25-2.13(\mathrm{~m}, 4 \mathrm{H})$ 2.02 (quintet, $J=6.4 \mathrm{~Hz}, 2 \mathrm{H}), 1.57-1.38(\mathrm{~m}, 4 \mathrm{H}), 0.94(\mathrm{t}, J=7.1$ $\mathrm{Hz}, 3 \mathrm{H}) ;{ }^{13} \mathrm{C}$ NMR $\left(75 \mathrm{MHz}, \mathrm{CDCl}_{3}\right) \delta 184.4,138.8,135.5,132.6$, 125.7, 125.4, 121.8, 117.7, 109.8, 82.3, 77.8, 45.7, 31.1, 28.7, 22.0, 21.4, 18.4, 16.0, 13.6; HRMS (ESI-TOF) $\mathrm{m} / \mathrm{z}:[\mathrm{M}+\mathrm{H}]^{+}$calcd for $\mathrm{C}_{19} \mathrm{H}_{24} \mathrm{NO} 282.1858$; Found, 282.1861.

5-Methoxy-1-(non-4-yn-1-yl)-1H-indole-3-carbaldehyde (3c): 5-methoxy-1H-indole-3-carbaldehyde ( $600 \mathrm{mg}, 3.43 \mathrm{mmol})$, sodium iodide ( $771 \mathrm{mg}, 1.5$ equiv), cesium carbonate ( $1.67 \mathrm{~g}, 1.5$ equiv), 1 chloronon-4-yne (653 mg, 1.2 equiv) and acetonitrile $(6.0 \mathrm{~mL})$ were employed. Upon silica gel column chromatography purification using ethyl acetate in hexanes $(0-50 \%)$, compound 3c was isolated as a white solid (927 mg, 91\%). M.p. 61-63 ${ }^{\circ} \mathrm{C}$; FTIR (thin film, $\left.\mathrm{cm}^{-1}\right) 2955,2930,1652,1530,1389 ;{ }^{1} \mathrm{H} \mathrm{NMR}\left(300 \mathrm{MHz}, \mathrm{CDCl}_{3}\right) \delta$ $9.96(\mathrm{~s}, 1 \mathrm{H}), 7.80(\mathrm{~d}, J=2.5 \mathrm{~Hz}, 1 \mathrm{H}), 7.71(\mathrm{~s}, 1 \mathrm{H}), 7.30(\mathrm{~d}, J=8.9$ $\mathrm{Hz}, 1 \mathrm{H}), 6.97(\mathrm{dd}, J=8.9,2.5 \mathrm{~Hz}, 1 \mathrm{H}), 4.31(\mathrm{t}, J=6.7 \mathrm{~Hz}, 2 \mathrm{H})$, $3.90(\mathrm{~s}, 3 \mathrm{H}), 2.26-2.14(\mathrm{~m}, 4 \mathrm{H}), 2.02$ (quintet, $J=6.5 \mathrm{~Hz}, 2 \mathrm{H})$, $1.48(\mathrm{~m}, 4 \mathrm{H}), 0.94(\mathrm{t}, J=7.1 \mathrm{~Hz}, 3 \mathrm{H}) ;{ }^{13} \mathrm{C} \mathrm{NMR}\left(75 \mathrm{MHz}, \mathrm{CDCl}_{3}\right)$ $\delta 184.4,156.6,138.8,132.0,126.1,117.8,114.3,110.9,103.4$, $82.3,77.8,55.7,45.8,31.1,28.7,22.0,18.4,16.0,13.6$; HRMS (ESI-TOF) $\mathrm{m} / \mathrm{z}$ : $[\mathrm{M}+\mathrm{H}]^{+}$calcd for $\mathrm{C}_{19} \mathrm{H}_{24} \mathrm{NO}_{2}$ 298.1807; Found, 298.1809 .

5-Fluoro-1-(non-4-yn-1-yl)-1 $H$-indole-3-carbaldehyde (3d): 5 fluoro- $1 H$-indole-3-carbaldehyde $(500 \mathrm{mg}, 3.07 \mathrm{mmol})$, sodium iodide (690 mg, 1.5 equiv), cesium carbonate ( $1.50 \mathrm{~g}, 1.5$ equiv), 1 chloronon-4-yne (585 mg, 1.2 equiv) and acetonitrile $(5.0 \mathrm{ml})$ were employed. Upon silica gel column chromatography purification using ethyl acetate in hexanes (0-50\%), compound 3d was isolated as a yellow oil (791 mg, 91\%). FTIR (thin film, $\mathrm{cm}^{-1}$ ) 2955, 2930, $1655,1531,1392 ;{ }^{1} \mathrm{H}$ NMR $\left(300 \mathrm{MHz}, \mathrm{CDCl}_{3}\right) \delta 9.98(\mathrm{~s}, 1 \mathrm{H}), 7.99$ $(\mathrm{dd}, J=9.2,2.5 \mathrm{~Hz}, 1 \mathrm{H}), 7.78(\mathrm{~s}, 1 \mathrm{H}), 7.35(\mathrm{dd}, J=9.0,4.2 \mathrm{~Hz}$, $1 \mathrm{H}), 7.07(\mathrm{td}, J=9.0,3.8 \mathrm{~Hz}, 1 \mathrm{H}), 4.34(\mathrm{t}, J=6.7 \mathrm{~Hz}, 2 \mathrm{H}), 2.25-$ $2.14(\mathrm{~m}, 4 \mathrm{H}), 2.03$ (quintet, $J=6.5 \mathrm{~Hz}, 2 \mathrm{H}), 1.47(\mathrm{~m}, 4 \mathrm{H}), 0.94(\mathrm{t}, J$ $=7.2 \mathrm{~Hz}, 3 \mathrm{H}) ;{ }^{13} \mathrm{C} \mathrm{NMR}\left(75 \mathrm{MHz}, \mathrm{CDCl}_{3}\right) \delta 184.2,159.7(\mathrm{~d}, J=$ $319 \mathrm{~Hz}), 139.7,133.6,125.9(\mathrm{~d}, J=11.0 \mathrm{~Hz}), 117.8(\mathrm{~d}, J=4.5 \mathrm{~Hz})$, $112.2(\mathrm{~d}, J=26.4 \mathrm{~Hz}), 111.0(\mathrm{~d}, J=9.8 \mathrm{~Hz}), 107.4(\mathrm{~d}, J=24.6 \mathrm{~Hz})$, 82.4, 77.6, 45.9, 31.1, 28.6, 22.0, 18.4, 15.9, 13.6; HRMS (ESITOF) $\mathrm{m} / \mathrm{z}:[\mathrm{M}+\mathrm{H}]^{+}$calcd for $\mathrm{C}_{18} \mathrm{H}_{21} \mathrm{FNO}$ 286.1607; Found, 286.1616

5-Bromo-1-(non-4-yn-1-yl)-1H-indole-3-carbaldehyde (3e): 5bromo- $1 H$-indole-3-carbaldehyde $(500 \mathrm{mg}, 2.23 \mathrm{mmol})$, sodium iodide (501 mg, 1.5 equiv), cesium carbonate ( $1.09 \mathrm{~g}, 1.5$ equiv), 1 chloronon-4-yne (425 mg, 1.2 equiv) and acetonitrile $(5.0 \mathrm{~mL})$ were employed. Upon silica gel column chromatography purification using ethyl acetate in hexanes $(0-50 \%)$, compound $\mathbf{3 e}$ was isolated as a white solid $(678 \mathrm{mg}, 88 \%)$. M.p. $74-75^{\circ} \mathrm{C}$; FTIR (thin film, $\left.\mathrm{cm}^{-1}\right)$ 2955, 2931, 1662, 1531, 1465, 1401; ${ }^{1} \mathrm{H}$ NMR (300 MHz, $\left.\mathrm{CDCl}_{3}\right) \delta 9.98(\mathrm{~s}, 1 \mathrm{H}), 8.48(\mathrm{~d}, J=1.9 \mathrm{~Hz}, 1 \mathrm{H}), 7.75(\mathrm{~s}, 1 \mathrm{H}), 7.43$ $(\mathrm{dd}, J=8.7,1.9 \mathrm{~Hz}, 1 \mathrm{H}), 7.30(\mathrm{~d}, J=8.7 \mathrm{~Hz}, 1 \mathrm{H}), 4.33(\mathrm{t}, J=6.7$ $\mathrm{Hz}, 2 \mathrm{H}), 2.26-2.12(\mathrm{~m}, 4 \mathrm{H}), 2.02$ (quintet, $J=6.4 \mathrm{~Hz}, 2 \mathrm{H}), 1.55-$ $1.35(\mathrm{~m}, 4 \mathrm{H}), 0.94(\mathrm{t}, J=7.1 \mathrm{~Hz}, 3 \mathrm{H}) ;{ }^{13} \mathrm{C} \mathrm{NMR}\left(75 \mathrm{MHz}, \mathrm{CDCl}_{3}\right)$ $\delta 184.2,139.0,135.8,127.0,126.9,124.8,117.5,116.6,111.5$, 82.6, 77.5, 45.9, 31.1, 28.6, 22.0, 18.4, 16.0, 13.6; HRMS (ESITOF) $\mathrm{m} / \mathrm{z}:[\mathrm{M}+\mathrm{H}]^{+}$calcd for $\mathrm{C}_{18} \mathrm{H}_{21} \mathrm{BrNO}$ 346.0807; Found, 346.0821

6-Methoxy-1-(non-4-yn-1-yl)-1H-indole-3-carbaldehyde (3f): 6-methoxy-1H-indole-3-carbaldehyde $(296 \mathrm{mg}, 1.69 \mathrm{mmol}$,), sodium iodide (380 mg, 1.5 equiv), cesium carbonate (826 mg, 1.5 equiv), 1-chloronon-4-yne ( $268 \mathrm{mg}, 1.0$ equiv) and acetonitrile (3.4 $\mathrm{mL}$ ) were employed. Upon silica gel column chromatography purification using ethyl acetate in hexanes (0-30\%), compound $3 \mathbf{f}$ was isolated as a yellow solid (264 mg, 53\%); M.p. $38-40{ }^{\circ} \mathrm{C}$; FTIR (thin film, $\mathrm{cm}^{-1}$ ) 2928, 2858, 1656, 1623, 1578, 1523; ${ }^{1} \mathrm{H}$ NMR $\left(400 \mathrm{MHz}, \mathrm{CDCl}_{3}\right) \delta 9.94(\mathrm{~s}, 1 \mathrm{H}), 8.18(\mathrm{~d}, J=8.7 \mathrm{~Hz}, 1 \mathrm{H}), 7.66(\mathrm{~s}$, $1 \mathrm{H}), 6.96(\mathrm{dd}, J=8.7,2.3 \mathrm{~Hz}, 1 \mathrm{H}), 6.88(\mathrm{~d}, J=2.1 \mathrm{~Hz}, 1 \mathrm{H}), 4.29$ (t, $J=6.7 \mathrm{~Hz}, 2 \mathrm{H}), 3.89(\mathrm{~s}, 3 \mathrm{H}), 2.25-2.14(\mathrm{~m}, 5 \mathrm{H}), 2.10-1.96(\mathrm{~m}$, $2 \mathrm{H}), 1.54-1.38(\mathrm{~m}, 4 \mathrm{H}), 0.93(\mathrm{t}, J=7.2 \mathrm{~Hz}, 3 \mathrm{H}) ;{ }^{13} \mathrm{C}$ NMR $(101$ $\left.\mathrm{MHz}, \mathrm{CDCl}_{3}\right) \delta 184.6,157.7,138.3,123.1,119.6,118.4,112.0$, 94.2, 82.6, 77.9, 77.4, 55.9, 45.7, 31.3, 28.7, 22.2, 18.6, 16.2, 13.8; HRMS (ESI-TOF) m/z: $[\mathrm{M}+\mathrm{Na}]^{+}$calcd for $\mathrm{C}_{19} \mathrm{H}_{23} \mathrm{NO}_{2} \mathrm{Na}$ 320.1626; Found, 320.1621.
Methyl 3-formyl-1-(non-4-yn-1-yl)-1 $\boldsymbol{H}$-indole-6-carboxylate (3g): methyl 3-formyl-1 $H$-indole-6-carboxylate $(500 \mathrm{mg}, 2.46$ $\mathrm{mmol}$ ), sodium iodide (553 mg, 1.5 equiv), cesium carbonate (1.20 g, 1.5 equiv), 1-chloronon-4-yne (468 mg, 1.2 equiv) and acetonitrile $(5.0 \mathrm{~mL})$ were employed. Upon silica gel column chromatography purification using ethyl acetate in hexanes $(0-50 \%)$, compound $\mathbf{3 g}$ was isolated as a yellow oil $(578 \mathrm{mg}$, 72\%). FTIR (thin film, $\mathrm{cm}^{-1}$ ) 2953, 2932, 1711, 1658, 1617, 1529, 1434; ${ }^{1} \mathrm{H}$ NMR $\left(300 \mathrm{MHz}, \mathrm{CDCl}_{3}\right) \delta 10.04(\mathrm{~s}, 1 \mathrm{H}), 8.34(\mathrm{~d}, J=8.3 \mathrm{~Hz}, 1 \mathrm{H}), 8.18(\mathrm{~s}$, $1 \mathrm{H}), 8.00(\mathrm{dd}, J=8.4,1.4 \mathrm{~Hz}, 1 \mathrm{H}), 7.90(\mathrm{~s}, 1 \mathrm{H}), 4.42$ (t, $J=6.6 \mathrm{~Hz}$, $2 \mathrm{H}), 3.96(\mathrm{~s}, 3 \mathrm{H}), 2.20(\mathrm{td}, J=7.9,4.7 \mathrm{~Hz}, 4 \mathrm{H}), 2.07$ (quintet, $J=$ $6.6 \mathrm{~Hz}, 2 \mathrm{H}), 1.58-1.37(\mathrm{~m}, 4 \mathrm{H}), 0.94(\mathrm{t}, J=7.2 \mathrm{~Hz}, 3 \mathrm{H}) ;{ }^{13} \mathrm{C}$ NMR $\left(75 \mathrm{MHz}, \mathrm{CDCl}_{3}\right) \delta 184.2,167.2,140.7,136.6,128.8,125.5,123.7$, $121.5,117.9,112.2,82.5,77.5,52.0,45.8,31.0,28.7,22.0,18.3$, 15.9, 13.5; HRMS (ESI-TOF) m/z: $[\mathrm{M}+\mathrm{H}]^{+}$calcd for $\mathrm{C}_{20} \mathrm{H}_{24} \mathrm{NO}_{3}$ 326.1756; Found, 326.1764.

1-(6-Methoxyhex-4-yn-1-yl)-1H-indole-3-carbaldehyde (3h): $1 H$-indole-3-carbaldehyde $(1.22 \mathrm{~g}, 7.61 \mathrm{mmol})$, sodium iodide $(1.71$ $\mathrm{g}, 1.5$ equiv), cesium carbonate (3.72 g, 1.5 equiv), 6-chloro-1methoxyhex-2-yne ${ }^{19}$ (1.34 g, 1.2 equiv) and acetonitrile $(12.2 \mathrm{~mL})$ were employed. Upon silica gel column chromatography purification using ethyl acetate in hexanes $(0-50 \%)$, compound $\mathbf{3 h}$ was isolated as a yellow oil (1.83 g, 63\%). FTIR (thin film, $\left.\mathrm{cm}^{-1}\right) 2932$, 2820, 1654, 1530, 1388; ${ }^{1} \mathrm{H}$ NMR $\left(300 \mathrm{MHz}, \mathrm{CDCl}_{3}\right) \delta 10.02$ (s, $1 \mathrm{H}), 8.35-8.29(\mathrm{~m}, 1 \mathrm{H}), 7.77(\mathrm{~s}, 1 \mathrm{H}), 7.45-7.28(\mathrm{~m}, 3 \mathrm{H}), 4.36(\mathrm{t}$, $J=6.7 \mathrm{~Hz}, 2 \mathrm{H}), 4.12(\mathrm{t}, J=2.1 \mathrm{~Hz}, 2 \mathrm{H}), 3.40(\mathrm{~s}, 3 \mathrm{H}), 2.29-2.24$ $(\mathrm{m}, 2 \mathrm{H}), 2.10$ (quintet, $J=6.6 \mathrm{~Hz}, 2 \mathrm{H}) ;{ }^{13} \mathrm{C} \mathrm{NMR}\left(75 \mathrm{MHz}, \mathrm{CDCl}_{3}\right)$ $\delta 184.4,139.1,137.1,125.2,123.9,122.7,121.8,117.9,110.2$, 84.7, 77.7, 59.9, 57.4, 45.5, 28.2, 15.9; HRMS (ESI-TOF) m/z: [M $+\mathrm{H}]^{+}$calcd for $\mathrm{C}_{16} \mathrm{H}_{18} \mathrm{NO}_{2}$ 256.1338; Found, 256.1338.

1-(8-((Tetrahydro-2H-pyran-2-yl)oxy)oct-4-yn-1-yl)-1 $H$ indole-3-carbaldehyde (3i): $1 H$-indole-3-carbaldehyde $(1.00 \mathrm{~g}$, $6.89 \mathrm{mmol})$, sodium iodide (1.55 g, 1.5 equiv), cesium carbonate (3.37 g, 1.5 equiv), 2-((8-chlorooct-4-yn-1-yl)oxy)tetrahydro- $2 H$ $\operatorname{pyran}^{20}(2.02 \mathrm{~g}, 1.2$ equiv) and acetonitrile $(9.0 \mathrm{~mL})$ were employed. Upon silica gel column chromatography purification using ethyl acetate in hexanes $(0-30 \%)$, compound $\mathbf{3 i}$ was isolated as a yellow oil (1.26 g, 52\%); FTIR (thin film, $\mathrm{cm}^{-1}$ ) 2942, 1662, 1616, 1533, 1468,$1401 ;{ }^{1} \mathrm{H}$ NMR $\left(400 \mathrm{MHz}, \mathrm{CDCl}_{3}\right) \delta 10.02(\mathrm{~s}, 1 \mathrm{H}), 8.35-$ $8.27(\mathrm{~m}, 1 \mathrm{H}), 7.78(\mathrm{~s}, 1 \mathrm{H}), 7.45-7.39(\mathrm{~m}, 1 \mathrm{H}), 7.37-7.28(\mathrm{~m}$, $2 \mathrm{H}), 4.60(\mathrm{dd}, J=4.3,2.8 \mathrm{~Hz}, 1 \mathrm{H}), 4.35(\mathrm{t}, J=6.7 \mathrm{~Hz}, 2 \mathrm{H}), 3.91-$ $3.82(\mathrm{~m}, 2 \mathrm{H}), 3.55-3.43(\mathrm{~m}, 2 \mathrm{H}), 2.33(\mathrm{tt}, J=7.1,2.4 \mathrm{~Hz}, 2 \mathrm{H})$, $2.22-2.13(\mathrm{~m}, 4 \mathrm{H}), 2.10-2.00(\mathrm{~m}, 2 \mathrm{H}), 1.88-1.77(\mathrm{~m}, 3 \mathrm{H}), 1.72$ $(\mathrm{tt}, J=9.1,3.2 \mathrm{~Hz}, 1 \mathrm{H}), 1.65-1.46(\mathrm{~m}, 4 \mathrm{H}) ;{ }^{13} \mathrm{C} \mathrm{NMR}(101 \mathrm{MHz}$, $\left.\mathrm{CDCl}_{3}\right) \delta 184.7,138.8,137.3,125.6,124.1,123.1,122.4,118.3$, $110.2,99.0,81.9,78.2,66.2,62.5,45.9,30.9,29.4,28.7,25.6,19.7$, 16.2, 15.8; HRMS (ESI-TOF) m/z: $[\mathrm{M}+\mathrm{H}]^{+}$calcd for $\mathrm{C}_{22} \mathrm{H}_{28} \mathrm{NO}_{3}$ 354.2064; Found, 354.2045.

1-(5-Phenylpent-4-yn-1-yl)-1 $H$-indole-3-carbaldehyde $\quad(3 \mathbf{j})$ : $1 H$-indole-3-carbaldehyde $(200 \mathrm{mg}, 1.38 \mathrm{mmol})$, sodium iodide (310 mg, 1.5 equiv), cesium carbonate (674 mg, 1.5 equiv), (5chloropent-1-yn-1-yl)benzene ${ }^{7 a, 21}$ (296 mg, 1.2 equiv) and acetonitrile $(2.0 \mathrm{~mL})$ were employed. Upon silica gel column chromatography purification using ethyl acetate in hexanes $(0-50 \%)$, compound $\mathbf{3} \mathbf{j}$ was isolated as a yellow oil $(322 \mathrm{mg}, 81 \%)$. FTIR (thin film, $\left.\mathrm{cm}^{-1}\right)$ 3103, 3052, 2942, 2809, 2754, 1654, 1530, 1467; ${ }^{1} \mathrm{H}$ NMR $\left(300 \mathrm{MHz}, \mathrm{CDCl}_{3}\right) \delta 10.02(\mathrm{~s}, 1 \mathrm{H}), 8.36-8.30(\mathrm{~m}, 1 \mathrm{H}), 7.80$ $(\mathrm{s}, 1 \mathrm{H}), 7.48-7.41(\mathrm{~m}, 3 \mathrm{H}), 7.38-7.30(\mathrm{~m}, 5 \mathrm{H}), 4.43(\mathrm{t}, J=6.7$ $\mathrm{Hz}, 2 \mathrm{H}), 2.45(\mathrm{t}, J=6.6 \mathrm{~Hz}, 2 \mathrm{H}), 2.19(\mathrm{p}, J=6.6 \mathrm{~Hz}, 2 \mathrm{H}) ;{ }^{13} \mathrm{C}$ NMR $\left(75 \mathrm{MHz}, \mathrm{CDCl}_{3}\right) \delta 184.5,138.6,137.2,131.6,128.4,128.1$, $125.5,124.0,123.3,123.0,122.2,118.2,110.1,87.6,82.5,45.7$, 28.4, 16.7; HRMS (ESI-TOF) m/z: $[\mathrm{M}+\mathrm{H}]^{+}$calcd for $\mathrm{C}_{20} \mathrm{H}_{18} \mathrm{NO}$ 288.1388; Found, 288.1399.

1-(8-Phenyloct-4-yn-1-yl)-1 H-indole-3-carbaldehyde (3k): $1 H$ indole-3-carbaldehyde (1.07 g, $7.36 \mathrm{mmol})$, sodium iodide (2.00 g, 1.5 equiv), cesium carbonate ( $4.33 \mathrm{~g}, 1.5$ equiv), (8-chlorooct-4-yn1-yl)benzene (1.95 g, 1.2 equiv) and acetonitrile $(10.0 \mathrm{~mL})$ were employed. Upon silica gel column chromatography purification using ethyl acetate in hexanes (0-30\%), compound $\mathbf{3 k}$ was isolated as a yellow oil (1.82 g, 75\%); FTIR (thin film, $\mathrm{cm}^{-1}$ ) 3025, 2935, $1660,1614,1576,1531 ;{ }^{1} \mathrm{H}$ NMR $\left(400 \mathrm{MHz}, \mathrm{CDCl}_{3}\right) \delta 10.01(\mathrm{~s}$, 1H), $8.36-8.29$ (m, 1H), 7.76 (s, 1H), $7.46-7.39$ (m, 1H), $7.37-$ 
$7.27(\mathrm{~m}, 4 \mathrm{H}), 7.24-7.17(\mathrm{~m}, 3 \mathrm{H}), 4.35(\mathrm{t}, J=6.7 \mathrm{~Hz}, 2 \mathrm{H}), 2.75$ $(\mathrm{dd}, J=8.3,6.9 \mathrm{~Hz}, 2 \mathrm{H}), 2.28-2.16(\mathrm{~m}, 4 \mathrm{H}), 2.11-2.01(\mathrm{~m}, 2 \mathrm{H})$, $1.92-1.81(\mathrm{~m}, 2 \mathrm{H}) ;{ }^{13} \mathrm{C}$ NMR $\left(101 \mathrm{MHz}, \mathrm{CDCl}_{3}\right) \delta 184.7,141.7$, $138.7,137.3,128.6,128.5,126.1,125.6,124.1,123.1,122.3,118.3$, $110.2,82.0,78.4,45.9,35.1,30.7,28.8,18.4,16.2$; HRMS (ESITOF) $\mathrm{m} / \mathrm{z}:[\mathrm{M}+\mathrm{H}]^{+}$calcd for $\mathrm{C}_{23} \mathrm{H}_{24} \mathrm{NO}$ 330.1852; Found, 330.1867 .

1-(Non-4-yn-1-yl)-1H-pyrrole-3-carbaldehyde (3I): $\quad 1 H$ pyrrole-3-carbaldehyde $(500 \mathrm{mg}, 5.26 \mathrm{mmol})$, sodium iodide $(1.18$ g, 1.5 equiv), cesium carbonate (2.57 g, 1.5 equiv), 1-chloronon-4yne $(1.00 \mathrm{~g}, 1.2$ equiv) and acetonitrile $(5.0 \mathrm{~mL})$ were employed. Upon silica gel column chromatography purification using ethyl acetate in hexanes $(0-50 \%)$, compound $3 \mathrm{I}$ was isolated as a yellow oil (1.12 g, 98\%). FTIR (thin film, $\mathrm{cm}^{-1}$ ) 2955, 2931, 1664, 1532, 1397; ${ }^{1} \mathrm{H}$ NMR $\left(300 \mathrm{MHz}, \mathrm{CDCl}_{3}\right) \delta 9.74(\mathrm{~s}, 1 \mathrm{H}), 7.30(\mathrm{t}, J=1.8$ $\mathrm{Hz}, 1 \mathrm{H}), 6.71-6.60(\mathrm{~m}, 2 \mathrm{H}), 4.06(\mathrm{t}, J=6.7 \mathrm{~Hz}, 2 \mathrm{H}), 2.22-2.12$ (m, 4H), 1.93 (quintet, $J=6.6 \mathrm{~Hz}, 2 \mathrm{H}), 1.54-1.35(\mathrm{~m}, 4 \mathrm{H}), 0.93(\mathrm{t}$, $J=7.1 \mathrm{~Hz}, 3 \mathrm{H}) ;{ }^{13} \mathrm{C}$ NMR $\left(75 \mathrm{MHz}, \mathrm{CDCl}_{3}\right) \delta 185.1,129.2,126.4$, 123.4, 108.1, 82.0, 77.7, 48.5, 31.0, 30.0, 21.9, 18.3, 15.7, 13.5; HRMS (ESI-TOF) m/z: $[\mathrm{M}+\mathrm{H}]^{+}$calcd for $\mathrm{C}_{14} \mathrm{H}_{20} \mathrm{NO} 218.1545$; Found, 218.1542 .

Methyl 4-formyl-1-(non-4-yn-1-yl)-1 $H$-pyrrole-2-carboxylate (3m): methyl 4-formyl-1H-pyrrole-2-carboxylate $(500 \mathrm{mg}, 3.27$ $\mathrm{mmol})$, sodium iodide $(735 \mathrm{mg}, 1.5$ equiv), cesium carbonate $(1.60$ g, 1.5 equiv), 1-chloronon-4-yne (623 mg, 1.2 equiv) and acetonitrile $(5.0 \mathrm{~mL})$ were employed. Upon silica gel column chromatography purification using ethyl acetate in hexanes $(0-50 \%)$, compound $3 \mathrm{~m}$ was isolated as a yellow oil $(857 \mathrm{mg}, 95 \%)$. FTIR (thin film, $\left.\mathrm{cm}^{-1}\right) 2955,2932,2861,1713,1675,1546,1419 ;{ }^{1} \mathrm{H}$ NMR $\left(300 \mathrm{MHz}, \mathrm{CDCl}_{3}\right) \delta 9.78(\mathrm{~s}, 1 \mathrm{H}), 7.50(\mathrm{~d}, J=1.9 \mathrm{~Hz}, 1 \mathrm{H}), 7.38(\mathrm{~d}$, $J=1.9 \mathrm{~Hz}, 1 \mathrm{H}), 4.48(\mathrm{t}, J=6.7 \mathrm{~Hz}, 2 \mathrm{H}), 3.84(\mathrm{~s}, 3 \mathrm{H}), 2.22-2.10$ $(\mathrm{m}, 4 \mathrm{H}), 1.96$ (quintet, $J=6.6 \mathrm{~Hz}, 2 \mathrm{H}), 1.46(\mathrm{~m}, 4 \mathrm{H}), 0.93(\mathrm{t}, J=$ $7.1 \mathrm{~Hz}, 3 \mathrm{H}) ;{ }^{13} \mathrm{C}$ NMR $\left(75 \mathrm{MHz}, \mathrm{CDCl}_{3}\right) \delta 185.0,160.8,133.5$, $124.5,123.7,117.7,81.9,77.9,51.4,48.7,31.0,29.8,21.9,18.3$, 15.6, 13.5; HRMS (ESI-TOF) m/z: $[\mathrm{M}+\mathrm{H}]^{+}$calcd for $\mathrm{C}_{16} \mathrm{H}_{22} \mathrm{NO}_{3}$ 276.1600; Found, 276.1610.

\section{Synthesis of Isocanthines}

To a 4-dram vial was added indole-3-aldehyde $3(0.50 \mathrm{mmol})$, in tert-butyl amine $(3.0 \mathrm{~mL})$. The vial was then sealed with a Teflonlined cap, and the mixture was heated to $80{ }^{\circ} \mathrm{C}$ for $2 \mathrm{~h}$. After completion of imine formation ( $>99 \%$ conversion observed by ${ }^{1} \mathrm{H}$ NMR), the reaction mixture was cooled to $23{ }^{\circ} \mathrm{C}$, and concentrated in vacuum. Catalyst $\left[\mathrm{Cp} * \mathrm{Rh}(\mathrm{MeCN})_{3}\right]\left(\mathrm{SbF}_{6}\right)_{2}(\mathbf{C 1}, 2.5 \mathrm{~mol} \%, 10.6$ $\mathrm{mg}$ ), oxidant $\mathrm{Cu}(\mathrm{OAc})_{2}$ (2.1 equiv, $197 \mathrm{mg}$ ) and dichloroethane (DCE, $3.0 \mathrm{~mL}$ ) were then added. The mixture was heated to $100{ }^{\circ} \mathrm{C}$ for $16 \mathrm{~h}$. The mixture was then cooled to $23{ }^{\circ} \mathrm{C}$ and quenched with 3 $\mathrm{mL}$ of saturated aqueous $\mathrm{NaHCO}_{3}$ solution. The aqueous layer was extracted with dichloromethane $(5 \mathrm{~mL} \times 3)$ and the organic layers were combined and concentrated in vacuum. The residue was then purified using column chromatography to give the desired isocanthine 1.

3-n-Butyl-5,6-dihydro-4H-indolo[3,2,1-ij][1,6]naphthyridine

(1a): 1-(non-4-yn-1-yl)-1H-indole-3-carbaldehyde (134 mg, 0.50 mmol), tert-butylamine $(3.0 \mathrm{~mL}),\left[\mathrm{Cp} * \mathrm{Rh}(\mathrm{MeCN})_{3}\right]\left(\mathrm{SbF}_{6}\right)_{2}(\mathbf{C 1}$, $10.6 \mathrm{mg}, 2.5 \mathrm{~mol} \%), \mathrm{Cu}(\mathrm{OAc})_{2}$ (197 mg, 2.1 equiv) and dichloroethane $(3.0 \mathrm{~mL})$ were employed. Upon silica gel column chromatography purification using $\mathrm{MeOH}$ in DCM gradient elution $(0$ $10 \%)$, compound 1a was isolated as a yellow solid (97 $\mathrm{mg}, 73 \%$ ). M.p. 61-63 ${ }^{\circ} \mathrm{C}$; FTIR (thin film, $\mathrm{cm}^{-1}$ ) 2951, 2927, 2857, 1604, 1442; ${ }^{1} \mathrm{H}$ NMR $\left(300 \mathrm{MHz}, \mathrm{CDCl}_{3}\right) \delta 9.07(\mathrm{~s}, 1 \mathrm{H}), 8.11$ (d, $J=7.8$ $\mathrm{Hz}, 1 \mathrm{H}), 7.48(\mathrm{td}, J=7.6,1.1 \mathrm{~Hz}, 1 \mathrm{H}), 7.38(\mathrm{~d}, J=8.1 \mathrm{~Hz}, 1 \mathrm{H})$, $7.32-7.24(\mathrm{~m}, 1 \mathrm{H}), 4.19(\mathrm{t}, J=5.8 \mathrm{~Hz}, 2 \mathrm{H}), 3.03(\mathrm{t}, J=6.2 \mathrm{~Hz}$, $2 \mathrm{H}$ ), $2.92(\mathrm{t}, J=7.9 \mathrm{~Hz}, 2 \mathrm{H}), 2.35$ (quintet, $J=6.0 \mathrm{~Hz}, 2 \mathrm{H}$ ), 1.84 $1.65(\mathrm{~m}, 2 \mathrm{H}), 1.44(\mathrm{dq}, J=14.6,7.3 \mathrm{~Hz}, 2 \mathrm{H}), 0.96(\mathrm{t}, J=7.3 \mathrm{~Hz}$, $3 \mathrm{H}) ;{ }^{13} \mathrm{C}$ NMR $\left(75 \mathrm{MHz}, \mathrm{CDCl}_{3}\right) \delta 153.0,142.9,140.1,139.5$, $125.9,121.5,121.0,120.0,116.0,113.1,108.6,40.4,34.1,32.1$, 22.8, 22.1, 21.5, 14.1; HRMS (ESI-TOF) $\mathrm{m} / \mathrm{z}:[\mathrm{M}+\mathrm{H}]^{+}$calcd for $\mathrm{C}_{18} \mathrm{H}_{21} \mathrm{~N}_{2}$ 265.1705; Found, 265.1717.

3-n-Butyl-10-methyl-5,6-dihydro-4H-indolo[3,2,1ij][1,6]naphthyridine (1b): 5-methyl-1-(non-4-yn-1-yl)- $1 H$-indole3-carbaldehyde $(141 \mathrm{mg}, 0.50 \mathrm{mmol})$, tert-butylamine $(3.0 \mathrm{~mL})$, $\left[\mathrm{Cp} * \mathrm{Rh}(\mathrm{MeCN})_{3}\right]\left(\mathrm{SbF}_{6}\right)_{2}(\mathbf{C 1}, 10.6 \mathrm{mg}, 2.5 \mathrm{~mol} \%), \mathrm{Cu}(\mathrm{OAc})_{2}(197$ $\mathrm{mg}, 2.1$ equiv) and dichloroethane $(3.0 \mathrm{~mL})$ were employed. Upon silica gel column chromatography purification using ethyl acetate in hexanes gradient elution ( $0-50 \%)$, compound $\mathbf{1 b}$ was isolated as a green solid (109 mg, 78\%). M.p. $76-78{ }^{\circ} \mathrm{C}$; FTIR (thin film, $\mathrm{cm}^{-1}$ ) 2951, 2930, 2858, 1632, 1482; ${ }^{1} \mathrm{H}$ NMR $\left(300 \mathrm{MHz}, \mathrm{CDCl}_{3}\right) 9.03$ (s, $1 \mathrm{H}), 7.59(\mathrm{~d}, J=2.4 \mathrm{~Hz}, 1 \mathrm{H}), 7.28(\mathrm{~d}, J=9.0 \mathrm{~Hz}, 1 \mathrm{H}), 7.11(\mathrm{dd}, J=$ $8.8,2.5 \mathrm{~Hz}, 1 \mathrm{H}), 4.15(\mathrm{t}, J=5.8 \mathrm{~Hz}, 2 \mathrm{H}), 3.93(\mathrm{~s}, 3 \mathrm{H}), 3.01(\mathrm{t}, J=$ $6.2 \mathrm{~Hz}, 2 \mathrm{H}$ ), 2.90 (t, $J=7.9 \mathrm{~Hz}, 2 \mathrm{H}$ ), 2.33 (quintet, $J=6.0 \mathrm{~Hz}, 2 \mathrm{H}$ ), $1.82-1.67(\mathrm{~m}, 2 \mathrm{H}), 1.44(\mathrm{dq}, J=14.6,7.3 \mathrm{~Hz}, 2 \mathrm{H}), 0.96(\mathrm{t}, J=7.3$ $\mathrm{Hz}, 3 \mathrm{H}) ;{ }^{13} \mathrm{C}$ NMR $\left(75 \mathrm{MHz}, \mathrm{CDCl}_{3}\right) \delta 154.3,152.9,143.3,139.7$, $135.2,122.0,116.0,114.9,113.0,109.2,104.1,56.1,40.5,34.2$, 32.1, 22.9, 22.2, 21.4, 14.1; HRMS (ESI-TOF) m/z: $[\mathrm{M}+\mathrm{H}]^{+}$calcd for $\mathrm{C}_{19} \mathrm{H}_{23} \mathrm{~N}_{2}$ 279.1861; Found, 279.1868 .

3-n-Butyl-10-methoxy-5,6-dihydro- $4 H$-indolo[3,2,1ij] [1,6]naphthyridine (1c): 5-methoxy-1-(non-4-yn-1-yl)- $1 \mathrm{H}$ indole-3-carbaldehyde (149 $\mathrm{mg}, 0.50 \mathrm{mmol})$, tert-butylamine $(3.0$ $\mathrm{mL}), \quad\left[\mathrm{Cp} * \mathrm{Rh}(\mathrm{MeCN})_{3}\right]\left(\mathrm{SbF}_{6}\right)_{2} \quad(\mathbf{C 1}, 10.6 \mathrm{mg}, 2.5 \mathrm{~mol} \%)$, $\mathrm{Cu}(\mathrm{OAc})_{2}(197 \mathrm{mg}, 2.1$ equiv) and dichloroethane $(3.0 \mathrm{~mL})$ were employed. Upon silica gel column chromatography purification using $\mathrm{MeOH}$ in $\mathrm{DCM}$ gradient elution $(0-10 \%)$, compound 1c was isolated as a white solid (111 mg, 75\%). M.p. $87-88^{\circ} \mathrm{C}$; FTIR (thin film, $\left.\mathrm{cm}^{-1}\right) 2951,2925,2857,1610,1572,1483 ;{ }^{1} \mathrm{H}$ NMR $(300$ $\left.\mathrm{MHz}, \mathrm{CDCl}_{3}\right) \delta 9.02(\mathrm{~s}, 1 \mathrm{H}), 7.90(\mathrm{~s}, 1 \mathrm{H}), 7.32-7.24(\mathrm{~m}, 2 \mathrm{H}), 4.15$ $(\mathrm{t}, J=5.8 \mathrm{~Hz}, 2 \mathrm{H}), 3.01(\mathrm{t}, J=6.2 \mathrm{~Hz}, 2 \mathrm{H}), 2.89(\mathrm{t}, J=7.9 \mathrm{~Hz}, 2 \mathrm{H})$, $2.53(\mathrm{~s}, 3 \mathrm{H}), 2.32$ (quintet, $J=6.0 \mathrm{~Hz}, 2 \mathrm{H}), 1.80-1.67(\mathrm{~m}, 2 \mathrm{H})$, 1.44 (dq, $J=14.6,7.3 \mathrm{~Hz}, 2 \mathrm{H}), 0.96(\mathrm{t}, J=7.3 \mathrm{~Hz}, 3 \mathrm{H}) ;{ }^{13} \mathrm{C} \mathrm{NMR}$ $\left(75 \mathrm{MHz}, \mathrm{CDCl}_{3}\right) \delta 152.9,143.1,139.6,138.5,129.3,127.1,121.7$, $121.1,115.8,113.0,108.2,40.4,34.2,32.2,22.9,22.2,21.5,21.4$, 14.1; HRMS (ESI-TOF) m/z: $[\mathrm{M}+\mathrm{H}]^{+}$calcd for $\mathrm{C}_{19} \mathrm{H}_{23} \mathrm{~N}_{2} \mathrm{O}$ 295.1810; Found, 295.1820.

3-n-Butyl-10-fluoro-5,6-dihydro- $4 H$-indolo $[3,2,1$ -

ij][1,6]naphthyridine (1d): 5-fluoro-1-(non-4-yn-1-yl)- $1 H$-indole3-carbaldehyde (143 mg, $0.50 \mathrm{mmol})$, tert-butylamine $(3.0 \mathrm{~mL})$, $\left[\mathrm{Cp} * \mathrm{Rh}(\mathrm{MeCN})_{3}\right]\left(\mathrm{SbF}_{6}\right)_{2}(\mathbf{C 1}, 10.6 \mathrm{mg}, 2.5 \mathrm{~mol} \%), \mathrm{Cu}(\mathrm{OAc})_{2}(197$ $\mathrm{mg}, 2.1$ equiv) and dichloroethane $(3.0 \mathrm{~mL})$ were employed. Upon silica gel column chromatography purification using ethyl acetate in hexanes gradient elution (0-50\%), compound 1d was isolated as a white solid (128 mg, 90\%). M.p. $101-103{ }^{\circ} \mathrm{C}$; FTIR (thin film, $\left.\mathrm{cm}^{-1}\right)$ 2952, 2929, 2959, 1633, 1572, 1478; ${ }^{1} \mathrm{H}$ NMR $(300 \mathrm{MHz}$, $\left.\mathrm{CDCl}_{3}\right) \delta 9.02(\mathrm{~s}, 1 \mathrm{H}), 7.76(\mathrm{dd}, J=8.9,2.4 \mathrm{~Hz}, 1 \mathrm{H}), 7.31-7.25$ $(\mathrm{m}, 1 \mathrm{H}), 7.20(\mathrm{td}, J=8.9,2.5 \mathrm{~Hz}, 1 \mathrm{H}), 4.16(\mathrm{t}, J=5.8 \mathrm{~Hz}, 2 \mathrm{H}), 3.01$ (t, $J=6.2 \mathrm{~Hz}, 2 \mathrm{H}), 2.90(\mathrm{t}, J=7.9 \mathrm{~Hz}, 2 \mathrm{H}), 2.34$ (quintet, $J=6.0$ $\mathrm{Hz}, 2 \mathrm{H}), 1.79-1.67(\mathrm{~m}, 4 \mathrm{H}), 1.44(\mathrm{dq}, J=14.6,7.3 \mathrm{~Hz}, 2 \mathrm{H}), 0.96$ $(\mathrm{t}, J=7.3 \mathrm{~Hz}, 3 \mathrm{H}) ;{ }^{13} \mathrm{C}$ NMR $\left(75 \mathrm{MHz}, \mathrm{CDCl}_{3}\right) \delta 157.8(\mathrm{~d}, J=316$ $\mathrm{Hz}), 153.5,143.6,140.0,136.5,122.0(\mathrm{~d}, J=9.8 \mathrm{~Hz}), 115.7(\mathrm{~d}, J=$ $4.1 \mathrm{~Hz}), 113.5(\mathrm{~d}, J=25.5 \mathrm{~Hz}), 108.9(\mathrm{~d}, J=9.2 \mathrm{~Hz}), 106.9(\mathrm{~d}, J=$ $24.2 \mathrm{~Hz}$ ), 40.5, 34.2, 32.1, 22.8, 22.1, 21.3, 14.1; HRMS (ESI-TOF) $\mathrm{m} / \mathrm{z}:[\mathrm{M}+\mathrm{H}]^{+}$calcd for $\mathrm{C}_{18} \mathrm{H}_{20} \mathrm{FN}_{2}$ 283.1611; Found, 283.1622 .

10-Bromo-3-n-butyl-5,6-dihydro-4H-indolo[3,2,1-

ij] [1,6]naphthyridine (1e): 5-bromo-1-(non-4-yn-1-yl)- $1 H$-indole3-carbaldehyde $(173 \mathrm{mg}, 0.50 \mathrm{mmol})$, tert-butylamine $(3.0 \mathrm{~mL})$, $\left[\mathrm{Cp} * \mathrm{Rh}(\mathrm{MeCN})_{3}\right]\left(\mathrm{SbF}_{6}\right)_{2}(\mathbf{C 1}, 10.6 \mathrm{mg}, 2.5 \mathrm{~mol} \%), \mathrm{Cu}(\mathrm{OAc})_{2}(197$ $\mathrm{mg}, 2.1$ equiv) and dichloroethane $(3.0 \mathrm{~mL})$ were employed. Upon silica gel column chromatography purification using ethyl acetate in hexanes gradient elution $(0-50 \%)$, compound 1e was isolated as a pale yellow solid (70 mg, 40\%); M.p. $96-98{ }^{\circ} \mathrm{C}$; FTIR (thin film, $\left.\mathrm{cm}^{-1}\right) 2945,2851,1628,1570,1450 ;{ }^{1} \mathrm{H}$ NMR $\left(400 \mathrm{MHz}, \mathrm{CDCl}_{3}\right) \delta$ $9.02(\mathrm{~d}, J=0.7 \mathrm{~Hz}, 1 \mathrm{H}), 8.21(\mathrm{dd}, J=2.0,0.5 \mathrm{~Hz}, 1 \mathrm{H}), 7.55(\mathrm{dd}, J$ $=8.6,1.9 \mathrm{~Hz}, 1 \mathrm{H}), 7.27-7.19(\mathrm{~m}, 1 \mathrm{H}), 4.23-4.05(\mathrm{~m}, 2 \mathrm{H}), 3.08-$ $2.95(\mathrm{~m}, 2 \mathrm{H}), 2.93-2.80(\mathrm{~m}, 2 \mathrm{H}), 2.41-2.24(\mathrm{~m}, 2 \mathrm{H}), 1.81-1.64$ $(\mathrm{m}, 3 \mathrm{H}), 1.44(\mathrm{dq}, J=14.8,7.3 \mathrm{~Hz}, 2 \mathrm{H}), 0.96(\mathrm{t}, J=7.3 \mathrm{~Hz}, 3 \mathrm{H})$; ${ }^{13} \mathrm{C}$ NMR $\left(101 \mathrm{MHz}, \mathrm{CDCl}_{3}\right) \delta 153.9,143.2,140.0,138.8,128.6$, $123.8,123.3,115.2,113.3,112.8,109.9,40.6,34.2,32.1,22.9$, 22.1, 21.4, 14.1; HRMS (ESI-TOF) $\mathrm{m} / \mathrm{z}:[\mathrm{M}+\mathrm{H}]^{+}$calcd for $\mathrm{C}_{18} \mathrm{H}_{20} \mathrm{BrN}_{2}$ 343.0810; Found, 343.0826.

\section{3-n-Butyl-9-methoxy-5,6-dihydro-4H-indolo[3,2,1-}

ij][1,6]naphthyridine (1f): 6-methoxy-1-(non-4-yn-1-yl)- $1 H$ indole-3-carbaldehyde (149 $\mathrm{mg}, 0.50 \mathrm{mmol})$, tert-butylamine $(3.0$ $\mathrm{mL}), \quad\left[\mathrm{Cp} * \mathrm{Rh}(\mathrm{MeCN})_{3}\right]\left(\mathrm{SbF}_{6}\right)_{2} \quad(\mathbf{C 1}, 10.6 \mathrm{mg}, 2.5 \mathrm{~mol} \%)$, $\mathrm{Cu}(\mathrm{OAc})_{2}(197 \mathrm{mg}, 2.1$ equiv) and dichloroethane $(3.0 \mathrm{~mL})$ were employed. Upon silica gel column chromatography purification using $\mathrm{MeOH}$ in $\mathrm{DCM}$ gradient elution (0-10\%), compound 1f was 
isolated as a white solid (115 mg, 78\%). M.p. $90-92{ }^{\circ} \mathrm{C}$; FTIR (thin film, $\left.\mathrm{cm}^{-1}\right)$ 2952, 2930, 2857, 1609, 1578, 1475; ${ }^{1} \mathrm{H}$ NMR $(300$ $\left.\mathrm{MHz}, \mathrm{CDCl}_{3}\right) \delta 8.96(\mathrm{~s}, 1 \mathrm{H}), 7.96(\mathrm{~d}, J=8.5 \mathrm{~Hz}, 1 \mathrm{H}), 6.90-6.83$ $(\mathrm{m}, 2 \mathrm{H}), 4.13(\mathrm{t}, J=5.8 \mathrm{~Hz}, 2 \mathrm{H}), 3.93(\mathrm{~s}, 3 \mathrm{H}), 3.01(\mathrm{t}, J=6.2 \mathrm{~Hz}$, 2H), 2.89 (t, $J=7.9 \mathrm{~Hz}, 2 \mathrm{H}$ ), 2.33 (quintet, $J=6.0 \mathrm{~Hz}, 2 \mathrm{H}$ ), 1.72 $(\mathrm{m}, 2 \mathrm{H}), 1.44(\mathrm{dq}, J=14.6,7.3 \mathrm{~Hz}, 2 \mathrm{H}), 0.96(\mathrm{t}, J=7.3 \mathrm{~Hz}, 3 \mathrm{H})$; ${ }^{13} \mathrm{C}$ NMR $\left(75 \mathrm{MHz}, \mathrm{CDCl}_{3}\right) \delta 159.2,152.3,143.2,141.6,138.7$, $121.7,116.2,115.2,112.9,108.1,93.2,55.7,40.5,34.2,32.2,22.9$, 22.2, 21.5, 14.1; HRMS (ESI-TOF) $\mathrm{m} / \mathrm{z}:[\mathrm{M}+\mathrm{H}]^{+}$calcd for $\mathrm{C}_{19} \mathrm{H}_{23} \mathrm{~N}_{2} \mathrm{O}$ 295.1810; Found, 295.1823.

Methyl 3-n-butyl-5,6-dihydro-4H-indolo[3,2,1ij][1,6]naphthyridine-9-carboxylate (1g): methyl 3-formyl-1-(non4-yn-1-yl)- $1 H$-indole-6-carboxylate $(163 \mathrm{mg}, 0.50 \mathrm{mmol})$, tertbutylamine $(3.0 \mathrm{~mL}),\left[\mathrm{Cp} * \mathrm{Rh}(\mathrm{MeCN})_{3}\right]\left(\mathrm{SbF}_{6}\right)_{2}(\mathbf{C 1}, 10.6 \mathrm{mg}, 2.5$ $\mathrm{mol} \%), \mathrm{Cu}(\mathrm{OAc})_{2}(197 \mathrm{mg}, 2.1$ equiv) and dichloroethane $(3.0 \mathrm{~mL})$ were employed. Upon silica gel column chromatography purification using $\mathrm{MeOH}$ in DCM gradient elution (0-10\%), compound $\mathbf{1 g}$ was isolated as a white solid $(133 \mathrm{mg}, 82 \%)$; M.p. $135-137{ }^{\circ} \mathrm{C}$; FTIR (thin film, $\mathrm{cm}^{-1}$ ) 3073, 2947, 2865, 2853, 1612, 1567, 1440; ${ }^{1} \mathrm{H}$ NMR (400 MHz, $\left.\mathrm{CDCl}_{3}\right) \delta 9.11(\mathrm{~s}, 1 \mathrm{H}), 8.18-8.07(\mathrm{~m}, 2 \mathrm{H})$, $7.98(\mathrm{dd}, J=8.1,1.5 \mathrm{~Hz}, 1 \mathrm{H}), 4.25(\mathrm{dd}, J=6.3,5.3 \mathrm{~Hz}, 2 \mathrm{H}), 3.99$ $(\mathrm{s}, 3 \mathrm{H}), 3.05(\mathrm{t}, J=6.2 \mathrm{~Hz}, 2 \mathrm{H}), 2.98-2.81(\mathrm{~m}, 2 \mathrm{H}), 2.43-2.29$ $(\mathrm{m}, 2 \mathrm{H}), 1.80-1.69(\mathrm{~m}, 2 \mathrm{H}), 1.44$ (sextet, $J=7.4 \mathrm{~Hz}, 2 \mathrm{H}), 0.97$ (t, $J=7.4 \mathrm{~Hz}, 3 \mathrm{H}) ;{ }^{13} \mathrm{C}$ NMR $\left(101 \mathrm{MHz}, \mathrm{CDCl}_{3}\right) \delta 167.6,154.3$, 144.2, 140.7, 139.7, 127.4, 125.5, 121.3, 120.7, 115.5, 113.4, 110.4, 52.3, 40.7, 34.2, 32.1, 22.8, 22.1, 21.5, 14.1; HRMS (ESI-TOF) $\mathrm{m} / \mathrm{z}:[\mathrm{M}+\mathrm{H}]^{+}$calcd for $\mathrm{C}_{20} \mathrm{H}_{23} \mathrm{~N}_{2} \mathrm{O}_{2}$ 323.1760; Found, 323.1770.

3-(Methoxymethyl)-5,6-dihydro-4H-indolo[3,2,1-

ij] [1,6]naphthyridine (1h): 1-(6-methoxyhex-4-yn-1-yl)-1Hindole-3-carbaldehyde $(128 \mathrm{mg}, 0.50 \mathrm{mmol})$, tert-butylamine $(3.0$ $\mathrm{mL}), \quad\left[\mathrm{Cp} * \mathrm{Rh}(\mathrm{MeCN})_{3}\right]\left(\mathrm{SbF}_{6}\right)_{2} \quad(\mathbf{C 1}, \quad 10.6 \mathrm{mg}, \quad 2.5 \mathrm{~mol} \%)$, $\mathrm{Cu}(\mathrm{OAc})_{2}$ (197 mg, 2.1 equiv) and dichloroethane $(3.0 \mathrm{~mL})$ were employed. Upon silica gel column chromatography purification using $\mathrm{MeOH}$ in DCM gradient elution (0-10\%), compound $\mathbf{1 h}$ was isolated as a yellow solid (83 mg, 66\%). M.p. $97-99{ }^{\circ}$ C; FTIR (thin film, $\left.\mathrm{cm}^{-1}\right)$ 2915, 2881, 2801, 1571, 1442; ${ }^{1} \mathrm{H}$ NMR $(400 \mathrm{MHz}$, $\left.\mathrm{CDCl}_{3}\right) \delta 9.11(\mathrm{~s}, 1 \mathrm{H}), 8.14(\mathrm{dt}, J=7.8,1.0 \mathrm{~Hz}, 1 \mathrm{H}), 7.52(\mathrm{ddd}, J=$ $8.3,7.2,1.2 \mathrm{~Hz}, 1 \mathrm{H}), 7.42(\mathrm{dt}, J=8.2,0.9 \mathrm{~Hz}, 1 \mathrm{H}), 7.31$ (ddd, $J=$ 8.1, 7.2, $1.0 \mathrm{~Hz}, 1 \mathrm{H}), 4.76(\mathrm{~s}, 2 \mathrm{H}), 4.27-4.15(\mathrm{~m}, 2 \mathrm{H}), 3.46(\mathrm{~s}, 3 \mathrm{H})$, $3.15(\mathrm{t}, J=6.2 \mathrm{~Hz}, 2 \mathrm{H}), 2.43-2.27(\mathrm{~m}, 2 \mathrm{H}) ;{ }^{13} \mathrm{C} \mathrm{NMR}(101 \mathrm{MHz}$, $\left.\mathrm{CDCl}_{3}\right) \delta 147.7,142.9,140.4,139.6,126.4,121.5,121.3,120.3$, 117.4, 115.4, 108.8, 73.9, 58.4, 40.7, 22.1, 21.2; HRMS (ESI-TOF) $\mathrm{m} / \mathrm{z}:[\mathrm{M}+\mathrm{H}]^{+}$calcd for $\mathrm{C}_{16} \mathrm{H}_{17} \mathrm{~N}_{2} \mathrm{O}$ 253.1341; Found, 253.1345 .

3-(3-((Tetrahydro-2H-pyran-2-yl)oxy)propyl)-5,6-dihydro4H-indolo[3,2,1-ij] [1,6]naphthyridine (1i): 1 -(8-((tetrahydro- $2 H$ pyran-2-yl)oxy)oct-4-yn-1-yl)-1 $H$-indole-3-carbaldehyde $(178 \mathrm{mg}$, $0.50 \mathrm{mmol})$, tert-butylamine $(3.0 \mathrm{~mL}),\left[\mathrm{Cp} * \mathrm{Rh}(\mathrm{MeCN})_{3}\right]\left(\mathrm{SbF}_{6}\right)_{2}$ (C1, $10.6 \mathrm{mg}, 2.5 \mathrm{~mol} \%), \mathrm{Cu}(\mathrm{OAc})_{2}(197 \mathrm{mg}, 2.1$ equiv) and dichloroethane $(3.0 \mathrm{~mL})$ were employed. Upon silica gel column chromatography purification using ethyl acetate in hexanes gradient elution $(0-50 \%)$, compound $\mathbf{1 i}$ was isolated as an orange oil (149 $\mathrm{mg}, 85 \%$ ). FTIR (thin film, $\mathrm{cm}^{-1}$ ) 2927, 1605, 1569, 1443, 1355, $1322 ;{ }^{1} \mathrm{H}$ NMR $\left(400 \mathrm{MHz}, \mathrm{CDCl}_{3}\right) \delta 9.07(\mathrm{~s}, 1 \mathrm{H}), 8.16-8.07(\mathrm{~m}$, 1H), 7.49 (ddd, $J=8.4,7.1,1.2 \mathrm{~Hz}, 1 \mathrm{H}), 7.39$ (dt, $J=8.0,0.9 \mathrm{~Hz}$, $1 \mathrm{H}), 7.33-7.28(\mathrm{~m}, 1 \mathrm{H}), 4.66-4.55(\mathrm{~m}, 1 \mathrm{H}), 4.26-4.17(\mathrm{~m}, 2 \mathrm{H})$, $3.95-3.78(\mathrm{~m}, 2 \mathrm{H}), 3.48$ (dt, $J=9.8,6.5 \mathrm{~Hz}, 2 \mathrm{H}), 3.12-2.94(\mathrm{~m}$, $4 \mathrm{H}), 2.34(\mathrm{~m}, 2 \mathrm{H}), 2.16-2.02(\mathrm{~m}, 2 \mathrm{H}), 1.79-1.47(\mathrm{~m}, 6 \mathrm{H}) ;{ }^{13} \mathrm{C}$ NMR $\left(101 \mathrm{MHz}, \mathrm{CDCl}_{3}\right) \delta 152.6,143.1,142.5,140.3,139.9,128.6$, $128.4,126.1,125.8,121.7,121.3,120.2,116.3,113.4,108.8,40.7$, 36.0, 34.1, 31.5, 22.3, 21.6; HRMS (ESI-TOF) m/z: $[\mathrm{M}+\mathrm{H}]^{+}$calcd for $\mathrm{C}_{22} \mathrm{H}_{27} \mathrm{~N}_{2} \mathrm{O}_{2}$ 351.2067; Found, 351.2054 .

3-(3-Phenylpropyl)-5,6-dihydro-4H-indolo[3,2,1-

ij][1,6]naphthyridine (1k): 1-(8-phenyloct-4-yn-1-yl)-1 $H$-indole-3carbaldehyde $(165 \mathrm{mg}, 0.50 \mathrm{mmol})$, tert-butylamine $(3.0 \mathrm{~mL})$, $\left[\mathrm{Cp} * \mathrm{Rh}(\mathrm{MeCN})_{3}\right]\left(\mathrm{SbF}_{6}\right)_{2}(\mathbf{C 1}, 10.6 \mathrm{mg}, 2.5 \mathrm{~mol} \%), \mathrm{Cu}(\mathrm{OAc})_{2}(197$ $\mathrm{mg}, 2.1$ equiv) and dichloroethane $(3.0 \mathrm{~mL})$ were employed. Upon silica gel column chromatography purification using ethyl acetate in hexanes gradient elution (0-50\%), compound $\mathbf{1 k}$ was isolated as an orange oil (142 mg, 87\%). FTIR (thin film, $\mathrm{cm}^{-1}$ ) 2930, 1604, 1570, 1497, 1444, 1321; ${ }^{1} \mathrm{H}$ NMR (400 MHz, $\left.\mathrm{CDCl}_{3}\right) \delta 9.10(\mathrm{~s}, 1 \mathrm{H}), 8.14$ $(\mathrm{dt}, J=7.8,1.0 \mathrm{~Hz}, 1 \mathrm{H}), 7.51(\mathrm{ddd}, J=8.3,7.2,1.2 \mathrm{~Hz}, 1 \mathrm{H}), 7.41$ (dt, $J=8.1,0.9 \mathrm{~Hz}, 1 \mathrm{H}), 7.34-7.27$ (m, 4H), $7.26-7.17(\mathrm{~m}, 3 \mathrm{H})$, $4.25-4.14(\mathrm{~m}, 2 \mathrm{H}), 3.02-2.93(\mathrm{~m}, 4 \mathrm{H}), 2.80-2.72(\mathrm{~m}, 2 \mathrm{H}), 2.34$ $(\mathrm{dq}, J=6.8,5.8 \mathrm{~Hz}, 2 \mathrm{H}), 2.19-2.08(\mathrm{~m}, 2 \mathrm{H}) ;{ }^{13} \mathrm{C} \mathrm{NMR}(101 \mathrm{MHz}$, $\left.\mathrm{CDCl}_{3}\right) \delta 152.6,143.1,142.5,140.3,139.9,128.6,128.4,126.1$, $125.8,121.7,121.3,120.2,116.3,113.4,108.8,40.7,36.0,34.1$, 31.5, 22.3, 21.6; HRMS (ESI-TOF) $\mathrm{m} / \mathrm{z}:[\mathrm{M}+\mathrm{H}]^{+}$calcd for $\mathrm{C}_{23} \mathrm{H}_{23} \mathrm{~N}_{2}$ 327.1856; Found, 327.1822.

1-n-Butyl-8,9-dihydro-7H-pyrrolo[3,2,1-ij] [1,6]naphthyridine (11): 1-(non-4-yn-1-yl)-1 $H$-pyrrole-3-carbaldehyde (109 mg, 0.50 mmol), tert-butylamine $(3.0 \mathrm{~mL}),\left[\mathrm{Cp} * \mathrm{Rh}(\mathrm{MeCN})_{3}\right]\left(\mathrm{SbF}_{6}\right)_{2}(\mathbf{C 1}$, $10.6 \mathrm{mg}, 2.5 \mathrm{~mol} \%), \mathrm{Cu}(\mathrm{OAc})_{2}(197 \mathrm{mg}, 2.1$ equiv) and dichloroethane $(3.0 \mathrm{~mL})$ were employed. Upon silica gel column chromatography purification using ethyl acetate in hexanes gradient elution $(0-50 \%)$, compound 11 was isolated as an orange oil (57 mg, $53 \%)$; FTIR (thin film, $\mathrm{cm}^{-1}$ ) 2951, 2928, 2869, 1670, 1619, 1460; ${ }^{1} \mathrm{H}$ NMR $\left(300 \mathrm{MHz}, \mathrm{CDCl}_{3}\right) \delta 8.71(\mathrm{~s}, 1 \mathrm{H}), 7.04(\mathrm{~d}, J=3.1 \mathrm{~Hz}, 1 \mathrm{H})$, $6.47(\mathrm{~d}, J=3.1 \mathrm{~Hz}, 1 \mathrm{H}), 4.12(\mathrm{t}, J=5.7 \mathrm{~Hz}, 2 \mathrm{H}), 2.95(\mathrm{t}, J=6.2 \mathrm{~Hz}$, $2 \mathrm{H}), 2.84(\mathrm{t}, J=7.8 \mathrm{~Hz}, 2 \mathrm{H}), 2.27$ (quintet, $J=6.0 \mathrm{~Hz}, 2 \mathrm{H}$ ), $1.79-$ $1.63(\mathrm{~m}, 2 \mathrm{H}), 1.41(\mathrm{dq}, J=14.6,7.3 \mathrm{~Hz}, 2 \mathrm{H}), 0.94(\mathrm{t}, J=7.3 \mathrm{~Hz}$, $3 \mathrm{H}) ;{ }^{13} \mathrm{C}$ NMR $\left(75 \mathrm{MHz}, \mathrm{CDCl}_{3}\right) \delta 148.1,140.2,138.9,126.9$, 121.4, 113.8, 99.9, 43.6, 33.5, 32.3, 22.8, 22.7, 21.3, 14.1; HRMS (ESI-TOF) m/z: $[\mathrm{M}+\mathrm{H}]^{+}$calcd for $\mathrm{C}_{14} \mathrm{H}_{19} \mathrm{~N}_{2}$ 215.1548; Found, 215.1556

Methyl

1-butyl-8,9-dihydro-7H-pyrrolo[3,2,1ij] [1,6]naphthyridine-5-carboxylate (1m): methyl 4-formyl-1(non-4-yn-1-yl)-1 $H$-pyrrole-2-carboxylate $(138 \mathrm{mg}, 0.50 \mathrm{mmol}$ ), tert-butylamine $(3.0 \mathrm{~mL}, 1.50 \mathrm{~mL} / \mathrm{mmol})$, $\left[\mathrm{Cp} * \mathrm{Rh}(\mathrm{MeCN})_{3}\right]\left(\mathrm{SbF}_{6}\right)_{2}$ (C1, $10.6 \mathrm{mg}, 2.50 \mathrm{~mol} \%), \mathrm{Cu}(\mathrm{OAc})_{2}(197 \mathrm{mg}, 2.1$ equiv) and dichloroethane $(3.0 \mathrm{~mL})$ were employed. Upon silica gel column chromatography purification using $\mathrm{MeOH}$ in $\mathrm{DCM}$ gradient elution (0-10\%), compound $1 \mathrm{~m}$ was isolated as a yellow solid $(105 \mathrm{mg}$, 71\%). M.p. $58-60{ }^{\circ} \mathrm{C}$; FTIR (thin film, $\mathrm{cm}^{-1}$ ) 2953, 2858, 1709 , 1614, 1436; ${ }^{1} \mathrm{H}$ NMR $\left(300 \mathrm{MHz}, \mathrm{CDCl}_{3}\right) \delta 8.80(\mathrm{~s}, 1 \mathrm{H}), 7.23(\mathrm{~s}$, $1 \mathrm{H}), 4.50$ (t, $J=5.8 \mathrm{~Hz}, 2 \mathrm{H}), 3.92(\mathrm{~s}, 3 \mathrm{H}), 2.94(\mathrm{t}, J=6.2 \mathrm{~Hz}, 2 \mathrm{H})$, $2.85(\mathrm{t}, J=7.8 \mathrm{~Hz}, 2 \mathrm{H}), 2.26$ (quintet, $J=6.0 \mathrm{~Hz}, 2 \mathrm{H}), 1.70(\mathrm{~m}$, $2 \mathrm{H}), 1.41(\mathrm{dq}, J=14.5,7.3 \mathrm{~Hz}, 2 \mathrm{H}), 0.95(\mathrm{t}, J=7.3 \mathrm{~Hz}, 3 \mathrm{H}) ;{ }^{13} \mathrm{C}$ NMR $\left(75 \mathrm{MHz}, \mathrm{CDCl}_{3}\right) \delta 162.2,150.6,142.7,140.8,127.8,119.7$, $114.5,108.4,51.7,43.7,33.8,32.1,22.8,22.8,21.2,14.0$; HRMS (ESI-TOF) m/z: $[\mathrm{M}+\mathrm{H}]^{+}$calcd for $\mathrm{C}_{16} \mathrm{H}_{21} \mathrm{~N}_{2} \mathrm{O}_{2}$ 273.1603; Found, 273.1610

Suzuki-Miyaura Coupling of Bromoisocanthine 1e

3-n-Butyl-10-cyclopropyl-5,6-dihydro-4H-indolo[3,2,1-

ij] [1,6]naphthyridine (4a): To a 2-dram vial was added bromoisocanthine 1e (86 mg, $0.25 \mathrm{mmol})$, potassium cyclopropyltrifluoroborate ( $45 \mathrm{mg}, 1.2$ equiv), palladium(II) acetate $(5.8 \mathrm{mg}, 10$ mol \%), di-(1-adamantyl)- $n$-butylphosphine (14.2 mg, $15 \mathrm{~mol} \%$ ), $\mathrm{Cs}_{2} \mathrm{CO}_{3}(245 \mathrm{mg}, 3.0$ equiv), $\mathrm{PhMe}(1.0 \mathrm{~mL})$ and water $(0.10 \mathrm{~mL})$. The mixture was vacuumed and backfilled with nitrogen $(\times 3)$ and heated to $100{ }^{\circ} \mathrm{C}$ for $16 \mathrm{~h}$. The mixture was cooled to $23{ }^{\circ} \mathrm{C}$, diluted with acetone $(10 \mathrm{~mL})$ and concentrated. The residue was purified by silica gel column chromatography using EtOAc in hexanes (0-80\%) as eluent to afford compound $\mathbf{4 a}$ as a pale yellow oil (32 $\mathrm{mg}, 42 \%$ ). FTIR (thin film, $\mathrm{cm}^{-1}$ ) 2954, 2927, 2857, 1610, 1575; ${ }^{1} \mathrm{H}$ NMR (400 $\left.\mathrm{MHz}, \mathrm{CDCl}_{3}\right) \delta 9.05(\mathrm{~d}, J=0.7 \mathrm{~Hz}, 1 \mathrm{H}), 7.82(\mathrm{dt}, J=1.5,0.7 \mathrm{~Hz}$, $1 \mathrm{H}), 7.30-7.26(\mathrm{~m}, 2 \mathrm{H}), 4.25-4.05(\mathrm{~m}, 2 \mathrm{H}), 3.06-2.98(\mathrm{~m}, 2 \mathrm{H})$, $2.98-2.86(\mathrm{~m}, 2 \mathrm{H}), 2.33(\mathrm{dq}, J=6.8,5.8 \mathrm{~Hz}, 2 \mathrm{H}), 2.16-2.02(\mathrm{~m}$, $1 \mathrm{H}), 1.80-1.70(\mathrm{~m}, 2 \mathrm{H}), 1.50-1.38(\mathrm{~m}, 2 \mathrm{H}), 1.04-0.99(\mathrm{~m}, 2 \mathrm{H})$, $0.96(\mathrm{t}, J=7.4 \mathrm{~Hz}, 3 \mathrm{H}), 0.80-0.74(\mathrm{~m}, 2 \mathrm{H}) ;{ }^{13} \mathrm{C} \mathrm{NMR}(101 \mathrm{MHz}$, $\left.\mathrm{CDCl}_{3}\right) \delta 143.4,139.0,138.8,124.9,121.7,118.3,115.9,113.2$, 108.4, 103.2, 40.6, 32.1, 29.7, 22.8, 22.1, 21.5, 15.5, 14.1, 9.0; HRMS (ESI-TOF) m/z: $[\mathrm{M}+\mathrm{H}]^{+}$calcd for $\mathrm{C}_{21} \mathrm{H}_{25} \mathrm{~N}_{2}$ 305.2018; Found, 305.2031.

3-Butyl-10-(furan-2-yl)-5,6-dihydro- $4 H$-indolo[3,2,1ij] [1,6] naphthyridine $\mathbf{( 4 b )}$ : To a 2-dram vial was added bromoisocanthine 1e $(50 \mathrm{mg}, 0.146 \mathrm{mmol})$, 2-furyl pinacol boronic ester (42 $\mathrm{mg}, \quad 1.5 \quad$ equiv), dichlorobis(di- $t$ butylphenylphosphine)palladium(II) $(4.8 \mathrm{mg}, 5 \mathrm{~mol} \%), \mathrm{K}_{3} \mathrm{PO}_{4} \bullet \mathrm{H}_{2} \mathrm{O}$ $(67 \mathrm{mg}, 2.0$ equiv), THF $(0.50 \mathrm{~mL})$ and water $(0.10 \mathrm{~mL})$. The mixture was vacuumed and backfilled with nitrogen $(\times 3)$ and heated to $65^{\circ} \mathrm{C}$ for $5 \mathrm{~h}$. The mixture was cooled to room temperature, diluted with acetone $(10 \mathrm{~mL})$ and concentrated. The residue was purified by silica gel column chromatography using EtOAc in hexanes (0-80\%) as eluent to afford compound $\mathbf{4 c}$ as a pale yellow solid $(38 \mathrm{mg}$, 
79\%). M.p. 95-96 ${ }^{\circ} \mathrm{C}$; FTIR (thin film, $\mathrm{cm}^{-1}$ ) 3073, 2947, 2865, $1612,1567,1440 ;{ }^{1} \mathrm{H}$ NMR $\left(300 \mathrm{MHz}, \mathrm{CDCl}_{3}\right) \delta 9.09(\mathrm{~s}, 1 \mathrm{H}), 8.40$ (d, $J=1.6 \mathrm{~Hz}, 1 \mathrm{H}), 7.79$ (dd, $J=8.5,1.7 \mathrm{~Hz}, 1 \mathrm{H}), 7.49(\mathrm{~d}, J=1.8$ $\mathrm{Hz}, 1 \mathrm{H}), 7.35(\mathrm{~d}, J=8.5 \mathrm{~Hz}, 1 \mathrm{H}), 6.67(\mathrm{dd}, J=3.4,0.8 \mathrm{~Hz}, 1 \mathrm{H})$, $6.51(\mathrm{dd}, J=3.3,1.8 \mathrm{~Hz}, 1 \mathrm{H}), 4.17(\mathrm{t}, J=5.8 \mathrm{~Hz}, 2 \mathrm{H}), 3.02(\mathrm{t}, J=$ $6.2 \mathrm{~Hz}, 2 \mathrm{H}), 2.96-2.77(\mathrm{~m}, 2 \mathrm{H}), 2.34(\mathrm{p}, J=6.0 \mathrm{~Hz}, 2 \mathrm{H}), 1.74(\mathrm{tt}$, $J=7.9,6.5 \mathrm{~Hz}, 2 \mathrm{H}), 1.45$ (sextet, $J=7.3 \mathrm{~Hz}, 2 \mathrm{H}), 0.97(\mathrm{t}, J=7.3$ $\mathrm{Hz}, 3 \mathrm{H}) .{ }^{13} \mathrm{C}$ NMR $\left(75 \mathrm{MHz}, \mathrm{CDCl}_{3}\right) \delta 154.8,153.6,143.4,141.4$, $140.0,139.5,123.6,122.4,121.9,116.5,116.1,113.2,111.7,108.8$, 103.6, 40.6, 34.3, 32.1, 22.9, 22.1, 21.5, 14.1; HRMS (ESI-TOF) $\mathrm{m} / \mathrm{z}$ : $[\mathrm{M}+\mathrm{H}]^{+}$calcd for $\mathrm{C}_{22} \mathrm{H}_{23} \mathrm{~N}_{2} \mathrm{O} 331.1810$; Found, 331.1819.

\section{ASSOCIATED CONTENT}

\section{Supporting Information}

General calculation information, copies of ${ }^{1} \mathrm{H}$ NMR and ${ }^{13} \mathrm{C}$ NMR of new compounds, and X-ray crystallographic data of $\mathbf{4 b}$. This material is available free of charge via the Internet at http://pubs.acs.org.

\section{AUTHOR INFORMATION}

\section{Corresponding Author}

*Email: fuyao@ustc.edu.cn; zhang.haiming@gene.com

\section{Notes}

Part of this research was presented in the Symposium of Applications of $\mathrm{C}-\mathrm{H}$ Functionalization, Pacifichem, Honolulu, $\mathrm{HI}$ in December, 2015. AYC was an undergraduate summer intern at Genentech Inc. where this work was initiated. The authors declare no competing financial interest.

\section{ACKNOWLEDGMENTS}

This paper is dedicated to Professor Richard C. Larock, Emeritus Professor of Chemistry at Iowa State University on the occasion of his 73rd birthday. The authors would like to thank Dr. Kevin Kou (University of California, Berkeley) for helpful discussion, Dr. Kenji Kurita (Genentech, Inc.) for collecting HRMS data, Mr. Malcolm Huestis (Genentech, Inc.) for providing catalyst $\mathbf{C 1}$, and Dr. Francis Gosselin (Genentech Inc.) for proof-reading the manuscript. AYC, RS and BMS are grateful to the NSF under the CCI Center for Selective C-H Functionalization (CHE-1205646 and CHE-1700982) for support.

\section{REFERENCES}

(1) Yanagisawa,H.; Shimoji,Y.; Hashimoto, T. Jpn. Kokai Tokkyo Koho, JP05310738, 1993, Heisei; Chem. Abstr. 1994, 120, 245056.

(2) Clark, R. D.; Miller, A. B.; Berger, J.; Repke, D. B.; Weinhardt, K. K.; Kowalczyk, B. A.; Eglen, R. M.; Bonhaus, D. W.; Lee, C.-H.; Michel, A. D.; Smith, W. L.; Wong, E. H. F. J. Med. Chem. 1993, 36, 2645.

(3) Costall, B., Naylor R. J. Curr Drug Targets CNS Neurol. Disord. 2004, 3, 27.

(4) (a) Gilchrist, T. L.; Kemmitt, P. D. Tetrahedron 1997, 53, 4447. (b) Naik, P. N.; Khan, A.; Kusurkar, R. S. Tetrahedron 2013, 69, 10733.

(5) For recent reviews on hetero-Diels-Alder cycloaddition, see: (a) Blond, G.; Gulea, M.; Mamane, V. Curr. Org. Chem. 2016, 20 , 2161. (b) Ishihara, K.; Sakakura, A. In Comprehensive Organic Synthesis, 2nd Ed.; Knochel, P.; Molander, G. A. Eds.; Elsevier, 2014, 5, 409 .

(6) (a) Snyder, S. A.; Vosburg, D. A.; Jarvis, M. G.; Markgraf, J. H.; Tetrahedron 2000, 56, 5329. (b) Naik, P. N.; Khan, A.; Kusurkar, R. S. Tetrahedron 2013, 69, 10733. (c) Eberle, M. K.; Shapiro, M. J.; Stucki, R. J. Org. Chem. 1987, 52, 4661.

(7) (a) Zhang, H.; Larock, R. C.; Org. Lett. 2002, 4, 3035. (b) Zhang, H.; Larock, R. C.; J. Org. Chem. 2003, 68, 5132.
(8) For recent reviews on $\mathrm{Rh}$-catalyzed $\mathrm{C}-\mathrm{H}$ functionalization, see: (a) Dong, Z.; Ren, Z.; Thompson, S. J.; Xu, Y.; Dong, G. Chem. Rev. 2017, 117, 9333. (b) Yang, Y.; Lan, J.; You, J. Chem. Rev. 2017, 117, 8787. (c) Ye, B.; Cramer, N. Acc. Chem. Res. 2015, 48, 1308. (d) Song, G.; Li, X. Acc. Chem. Res. 2015, 48, 1007. (e) Colby, D. A.; Tsai, A. S.; Bergman, R. G.; Ellman, J. A. Acc. Chem. Res. 2012, 45, 814 .

(9) (a) Liu, B.; Song, C.; Sun, C.; Zhou, S.; Zhu, J.; J. Am. Chem. Soc. 2013, 135, 16625. (b) Stuart, D. R.; Alsabeh, P.; Kuhn, M.; Fagnou, K. J. Am. Chem. Soc. 2010, 132, 18326.

(10) Guimond, N.; Fagnou, K. J. Am. Chem. Soc. 2009, 131, 12050.

(11) (a) Han, W.; Zhang, G.; Li, G.; Huang, H. Org. Lett. 2014, 16, 3532. (b) Kim, D.; Park, J.; Jun, C. Adv. Synth. Catal. 2013, 355, 2667. (c) Shi, Z; Koester, D. C.; Boultadakis-Arapinis, M.; Glorius, F. J. Am. Chem. Soc. 2013, 135, 12204. (d) Zheng, L.; Ju, J.; Bin, Y.; Hua, R. J. Org. Chem. 2012, 77, 5794. (e) Zhang, X.; Chen, D.; Zhao, M.; Zhao, J.; Jia, A.; Li, X. Adv. Synth. Catal. 2011, 353, 719. (f) Too, P. C.; Chua, S. H.; Wong, S. H.; Chiba, S. J. Org. Chem. 2011, 76, 6159. (g) Too, P. C.; Wang, Y.-F.; Chiba, S. Org. Lett. 2010, 12, 5688. (h) Parthasarathy, K.; Cheng, C. H. J. Org. Chem. 2009, 74, 9359. (i) Colby, D. A.; Bergman, R. G.; Ellman, J. A. J. Am. Chem. Soc. 2008. 130, 3645.

(12) (a) Guimond, N.; Gorelsky, S.; Fagnou, K. J. Am. Chem Soc. 2011, 133, 6449. (b) Guimond, N.; Gouliaras, C.; Fagnou, K. J. Am. Chem. Soc. 2010, 132, 6908. (c) Hyster, T. K.; Rovis, T. Chem. Commun. 2011, 47, 11846. (d) Hyster, T. K.; Rovis, T. J. Am. Chem. Soc. 2010, 132, 10565. (e) Hyster. T. K.; Rovis, T. Synlett 2013, 24, 1842. (f) Prakash, P.; Jijy, E.; Aparna, P. S.; Radharkrishnan, K. V. Tetrahedron Lett. 2014, 55, 916.

(13) Lian, Y.; Huber, T.; Hesp, K. D.; Bergman, R.G.; Ellman, J. A. Angew. Chem., Int. Ed. 2013, 52. 629.

(14) (a) Martin, R. M.; Bergman, R. G.; Ellman, J. A. J. Org Chem. 2012, 77, 2501.

(15) Zheng, L.; Bin, Y.; Wang, Y.; Hua, R. J. Org. Chem. 2016, 81,8911 . Two examples of 1-methylisocanthines were reported in this study. We initially attempted the $\mathrm{C}-\mathrm{H}$ functionalization of aldehyde 3a under conditions described in ref. 15, however, no significant amount of isocanthine 1a was observed.

(16) (a) Huestis, M. P. J. Org. Chem. 2016, 81, 12545. (b) Huestis, M. P.; Chan, L.; Stuart, D. R.; Fagnou, K. Angew. Chem., Int. Ed. 2011, 50, 1338.

(17) Further investigations on this reaction are underway. Coincidentally, no phenyl-substituted alkynes were reported in Fagnou's isoquinoline synthesis, see ref. 10.

(18) For reviews on Suzuki-Miyaura coupling, see: (a) Miyaura, N.; Suzuki, A. Chem. Rev. 1995, 95, 2457. (b) Hassan, J.; Sévignon, M.; Gozzi, C.; Schulz, E.; Lemaire, M. Chem. Rev. 2002, 102, 1359.

(19) Massardo, P.; Bettarini, F.; Bianchini, E.; Piccardi, P. Gen. Offen. 1983, 3307614

(20) Ireland, R. E.; Brown Jr., F. R. J. Org. Chem. 1980, 45, 1868

(21) Baker, M. V.; Brown, D. H.; Skelton, B. W.; White, A. H. J. Chem. Soc., Dalton Trans. 2000, 4607. 